\title{
Canonisation and Definability for Graphs of Bounded Rank Width
}

\author{
Martin Grohe \\ RWTH Aachen University \\ grohe@informatik.rwth-aachen.de
}

\author{
Daniel Neuen \\ Simon Fraser University \\ dneuen@sfu.ca
}

\begin{abstract}
We prove that the combinatorial Weisfeiler-Leman algorithm of dimension $(3 k+4)$ is a complete isomorphism test for the class of all graphs of rank width at most $k$. Rank width is a graph invariant that, similarly to tree width, measures the width of a certain style of hierarchical decomposition of graphs; it is equivalent to clique width.

It was known that isomorphism of graphs of rank width $k$ is decidable in polynomial time (Grohe and Schweitzer, FOCS 2015), but the best previously known algorithm has a running time $n^{f(k)}$ for a non-elementary function $f$. Our result yields an isomorphism test for graphs of rank width $k$ running in time $n^{O(k)}$. Another consequence of our result is the first polynomial-time canonisation algorithm for graphs of bounded rank width.

Our second main result is that fixed-point logic with counting captures polynomial time on all graph classes of bounded rank width.
\end{abstract}

\section{Introduction}

Rank width, introduced by Oum and Seymour [39, 41], is a graph invariant that measures how well a graph can be decomposed hierarchically in a certain style. In this respect, it is similar to the better-known tree width, but where tree width measures the complexity, or width, of a separation in such a hierarchical decomposition in terms of the "connectivity" between the two sides, rank width measures the complexity of a separation in terms of the rank of the adjacency matrix of the edges between the two sides of the separation. This makes rank width (almost) invariant under complementation of a graph and thus relevant for dense graphs, where tree width usually becomes meaningless. Rank width is closely related to clique width, which had been introduced by Courcelle and Olariu [11]: for every graph $G$ it holds that $\operatorname{rw}(G) \leq \operatorname{cw}(G) \leq$ $2^{\mathrm{rw}(G)+1}-1$, where $\operatorname{rw}(G)$ denotes the rank width and $\mathrm{cw}(G)$ the clique width of $G$. This implies that many hard algorithmic problems can be solved efficiently on graphs of bounded rank width (see, for example, [13]), among them all problems definable in monadic second-order logic [10]. Furthermore, graph classes of bounded clique width, or equivalently bounded rank width, are precisely those that can be obtained by means of a monadic second-order transduction over a class of trees $[7,8,9]$.

In this paper we study the graph isomorphism problem and the closely related graph canonisation problem as well as logical definability and descriptive complexity on graph classes of bounded rank width.

Despite Babai's quasipolynomial time algorithm [2], it is still wide open whether the graph isomorphism problem can be solved in polynomial time. Polynomial-time algorithms are only known for specific graph classes, among them all classes of bounded degree [37], all classes of bounded tree width [3,36], all classes excluding a fixed graph as a minor [42], even all classes excluding a fixed graph as a topological subgraph [22], and most recently, graph classes of bounded rank width [24]. This last result was the starting point for our present paper. The running time of the isomorphism test in [24] is $n^{f(k)}$, where $n$ is the number of vertices 
and $k$ the rank width of the input graph, and $f$ is a non-elementary function. Of course this is unsatisfactory. Moreover, the algorithm is extremely complicated, using both advanced techniques from structural graph theory $[43,41,25]$ and the group-theoretic graph isomorphism machinery [37].

Our first contribution is a simple isomorphism test for graphs of rank width at most $k$ running in time $n^{O(k)}$. Indeed, the algorithm we use is a generic combinatorial isomorphism test known as the Weisfeiler-Leman algorithm [45, 2, 5]. The $\ell$-dimensional Weisfeiler Leman algorithm ( $\ell$-WL) iteratively colours $\ell$-tuples of vertices of the two input graphs and then compares the resulting colour patterns. If they differ, we know that the two input graphs are nonisomorphic. If two graphs have the same colour pattern, in general they may still be nonisomorphic [5]. Thus, $\ell$-WL is not a complete isomorphism test for all graphs. However, we prove that it is for graphs of bounded rank width. We say that $\ell$-WL identifies a graph $G$ if it distinguishes $G$ from every graph $H$ not isomorphic to $G$.

Theorem 1.1. The $(3 k+4)$-dimensional Weisfeiler-Leman algorithm identifies every graph of rank width at most $k$.

Combining this theorem with a result due to Immerman and Lander on the running time of the WL algorithm, we obtain the following.

Corollary 1.2. Isomorphism of graphs of rank width $k$ can be decided in time $O\left(n^{3 k+5} \log n\right)$.

Another way of stating Theorem 1.1 is that the Weisfeiler-Leman (WL) dimension [19] of graphs of rank width $k$ is at most $3 k+4$. While it is known that many natural graph classes have bounded WL dimension, among them the class of planar graphs [17,33], classes of bounded genus [18, 20], bounded tree width [21,32], classes of graphs excluding some fixed graph as a minor [19], and interval graphs [14], all these except for the class of interval graphs are classes of sparse graphs (with an edge number linear in the number of vertices). Our result adds a rich family of classes that include dense graphs to the picture.

Immerman and Lander [31] (also see [5]) showed that $\ell$-WL is an equivalence test for $C^{\ell+1}$, the $(\ell+1)$-variable fragment of first-order logic with counting. Hence our result can also be read as a definability result.

Corollary 1.3. For every graph $G$ of rank width at most $k$ there is a sentence $\varphi_{G}$ of the logic $\mathrm{C}^{3 k+5}$ that characterises $G$ up to isomorphism.

We use this connection to logic in our proof of Theorem 1.1, which is based on a characterisation of equivalence in the logic $C^{\ell}$ in terms of an Ehrenfeucht-Fraïssé game, the so-called -bijective pebble game due to Hella [28].

A canonisation algorithm $A$ for a class $\mathcal{C}$ of graphs associates with each graph $G \in \mathcal{C}$ a graph $A(G)$ that is isomorphic to $G$ in such a way that if $G, H \in \mathcal{C}$ are isomorphic then $A(G)$ and $A(H)$ are identical. Clearly, a canonisation algorithm can be used to test if two graphs are isomorphic; the converse is not known. It is known, ${ }^{1}$ however, that if a class of graphs has WL dimension at most $\ell$ then there is a canonisation algorithm for this class running in time $O\left(n^{\ell+3} \log n\right)$. Hence, as another corollary to Theorem 1.1, we obtain the first polynomial-time canonisation algorithm for graphs of bounded rank width.

Corollary 1.4. There is a canonisation algorithm for the class of graphs of rank width at most $k$ running in time $O\left(n^{3 k+7} \log n\right)$.

The second part of our paper is concerned with descriptive complexity theory. The central open question of the field is whether there is a logic that captures polynomial time [6, 26].

\footnotetext{
${ }^{1}$ The result is certainly not new and not ours, but unfortunately we are not aware of a reference. We sketch a proof in Appendix A.
} 
Intuitively, this means that all sentences of the logic can be evaluated in polynomial time (by a uniform algorithm) and that all polynomial-time decidable properties can be defined in the logic. By the Immerman-Vardi Theorem [29, 44], least-fixed point logic LFP captures polynomial time on ordered structures (that is, structures with a distinguished binary relation that is a linear order of the universe). But for general structures the question is still wide open more than 35 years after it was first raised by Chandra and Harel [6]. The question is related to isomorphism testing and canonisation. Indeed, a polynomial-time canonisation algorithm for the class of all graphs would imply that there is a logic capturing polynomial time.

The question for a logic capturing polynomial time, as formulated by Gurevich [26], casts the notion of what constitutes a logic deliberately wide. However, we are not mainly interested in an abstract logic, but in a "nice" logic that conveys some insights on the nature of polynomialtime computation. A logic that arguably falls in this category is fixed-point logic with counting FP+C, first proposed by Immerman [29] and later formalised by Grädel and Otto [16] in the form commonly used today. It is known that $\mathrm{FP}+\mathrm{C}$ does not capture polynomial time [5]. But over the last 10 years it has become clear that the logic is surprisingly powerful. It captures specific polynomial time algorithms such as linear programming [1], and it does capture polynomial time on rich graph classes, including all classes excluding some fixed graph as a minor [19]. Our second main result further broadens the scope of FP+C-definability.

Theorem 1.5. For every $k$, fixed-point logic with counting FP+C captures polynomial time on the class of all graphs of rank width at most $k$.

Technically, this theorem is related to the first and is based on the same graph-theoretic ideas, but it is significantly harder to prove. On an abstract level, this can be explained by highlighting an important difference between Theorem 1.5 and Corollary 1.3, which rephrases Theorem 1.1 in terms of logic. Corollary 1.3 is a nonuniform definability result: for every fixed graph we construct a formula characterising this graph. By contrast, Theorem 1.5 requires uniform definability: for every polynomial time property we want a unique sentence that defines this property for all graphs of rank width at most $k$. This means that we have to internalise the construction that is underlying the proof of Theorem 1.1 in the logic FP+C.

The paper is organised as follows: after reviewing the necessary preliminaries on rank width, graph isomorphism testing, and the WL algorithm in Section 2, in Section 3 we introduce our technical machinery for dealing with rank decompositions that is underlying the proofs of both theorems. We prove Theorem 1.1 in Section 4 and Theorem 1.5 in Section 5, after giving additional background in descriptive complexity theory in Subsection 5.1.

\section{Preliminaries}

\subsection{Graphs}

A graph is a pair $G=(V, E)$ with vertex set $V=V(G)$ and edge relation $E=E(G)$. In this paper all graphs are finite, simple (no loops or multiple edges), and undirected. We denote edges by $v w \in E(G)$ where $v, w \in V(G)$. The neighbourhood of $v \in V(G)$ is denoted by $N(v)$. For $A \subseteq V(G)$ we denote by $G[A]$ the induced subgraph of $G$ on $A$. Also, we denote by $G \backslash A$ the induced subgraph on the complement of $A$, that is $G \backslash A:=G[V(G) \backslash A]$.

An isomorphism from a graph $G$ to another graph $H$ is a bijective mapping $\varphi: V(G) \rightarrow V(H)$ which preserves the edge relation, that is, $v w \in E(G)$ if and only if $\varphi(v) \varphi(w) \in E(H)$ for all $v, w \in V(G)$. Two graphs $G$ and $H$ are isomorphic $(G \cong H)$ if there is an isomorphism from $G$ to $H$. We write $\varphi: G \cong H$ to denote that $\varphi$ is an isomorphism from $G$ to $H$.

A (vertex-)coloured graph is a tuple $(G, \chi)$ where $\chi: V(G) \rightarrow \mathcal{C}$ is a mapping and $\mathcal{C}$ is a finite set of colours. Typically the set of colours is just an initial segment $[n]:=\{1, \ldots, n\}$ of the natural numbers. Isomorphisms between coloured graphs have to respect the colours of the 
vertices. In this paper, we typically consider coloured graphs also when not explicitly stated. Note that an uncoloured graph may be viewed as a coloured graph where each vertex gets the same colour.

\subsection{Rank Width and Clique Width}

In this work, we are interested in graphs of bounded rank width and graphs of bounded clique width. This section formally defines both parameters and describes the basic connections between them.

Rank Width Rank width is a graph invariant that was first introduced by Oum and Seymour [41] and which measures the width of a certain style of hierarchical decomposition of graphs. Intuitively, the aim is to repeatedly split the vertex set of the graph along cuts of low complexity in a hierarchical fashion. For rank width, the complexity of a cut is measured in terms of the rank of the matrix capturing the adjacencies between the two sides of the cut over the 2-element field $\mathbb{F}_{2}$.

Let $G$ be a graph. For $X, Y \subseteq V(G)$ we define $M(X, Y) \in \mathbb{F}_{2}^{X \times Y}$ where $(M(X, Y))_{x, y}=1$ if and only if $x y \in E(G)$. Furthermore $\rho_{G}(X):=\operatorname{rk}_{2}(M(X, \bar{X}))$ where $\bar{X}:=V(G) \backslash X$ and $\operatorname{rk}_{2}(A)$ denotes the $\mathbb{F}_{2}$-rank of a matrix $A$.

A rank decomposition of $G$ is a tuple $(T, \gamma)$ consisting of a binary rooted tree $T$ and a mapping $\gamma: V(T) \rightarrow 2^{V(G)}$ such that

(R.1) $\gamma(r)=V(G)$ where $r$ is the root of $T$,

(R.2) $\gamma(t)=\gamma\left(s_{1}\right) \cup \gamma\left(s_{2}\right)$ and $\gamma\left(s_{1}\right) \cap \gamma\left(s_{2}\right)=\emptyset$ for all internal nodes $t \in V(T)$ with children $s_{1}$ and $s_{2}$, and

(R.3) $|\gamma(t)|=1$ for all $t \in L(T)$, where $L(T)$ denotes the set of leaves of the tree $T$.

Note that, instead of giving $\gamma$, we can equivalently specify a bijection $f: L(T) \rightarrow V(G)$ (this completely specifies $\gamma$ by Condition (R.2)). The width of a rank decomposition $(T, \gamma)$ is

$$
\operatorname{wd}(T, \gamma):=\max \left\{\rho_{G}(\gamma(t)) \mid t \in V(T)\right\} .
$$

The rank width of a graph $G$ is

$$
\operatorname{rw}(G):=\min \{\operatorname{wd}(T, \gamma) \mid(T, \gamma) \text { is a rank decomposition of } G\} \text {. }
$$

Clique Width Clique width [11] is another measure aiming to describe the structural complexity of a graph, but unlike rank width, it considers the complexity of an algebraic expression defining the graph.

For $k \in \mathbb{N}$ a $k$-graph is a pair $(G$, lab) where $G$ is a graph and lab: $V(G) \rightarrow[k]$ is a labelling of vertices. We define the following four operations for $k$-graphs:

(1) for $i \in[k]$ let $\cdot_{i}$ denote an isolated vertex with label $i$,

(2) for $i, j \in[k]$ with $i \neq j$ we define $\eta_{i, j}(G$, lab $)=\left(G^{\prime}\right.$, lab) where $V\left(G^{\prime}\right):=V(G)$ and $E\left(G^{\prime}\right):=E(G) \cup\{v w \mid \operatorname{lab}(v)=i \wedge \operatorname{lab}(w)=j\}$,

(3) for $i, j \in[k]$ we define $\rho_{i \rightarrow j}(G$, lab $)=(G$, lab' $)$ where

$$
\operatorname{lab}^{\prime}(v):=\left\{\begin{array}{ll}
j & \text { if } \operatorname{lab}(v)=i \\
\operatorname{lab}(v) & \text { otherwise }
\end{array},\right.
$$


(4) for two $k$-graphs $\left(G\right.$, lab) and $\left(G^{\prime}\right.$, lab $)$ we define $\left(G\right.$, lab) $\oplus\left(G^{\prime}\right.$, lab' $)$ to be the disjoint union of the two $k$-graphs.

A $k$-expression $t$ is a well-formed expression in these symbols and defines a $k$-graph $(G$, lab). In this case $t$ is a $k$-expression for $G$. The clique width of a graph $G$, denoted by $\operatorname{cw}(G)$, is the minimum $k \in \mathbb{N}$ such that there is a $k$-expression for $G$.

Comparing clique width and rank width, each parameter is bounded in terms of the other.

Theorem 2.1 ([41]). For every graph $G$ it holds that

$$
\mathrm{rw}(G) \leq \mathrm{cw}(G) \leq 2^{\mathrm{rw}(G)+1}-1 .
$$

Also, there is the following connection to tree width.

Theorem 2.2 ([40]). For every graph $G$ it holds that

$$
\operatorname{rw}(G) \leq \operatorname{tw}(G)+1
$$

where $\operatorname{tw}(G)$ denotes the tree width of $G$.

Note that the tree width of a graph can not be bounded in terms of its rank width. For example, the complete graph on $n$ vertices $K_{n}$ has rank width $\operatorname{rw}\left(K_{n}\right)=1$ and tree width $\operatorname{tw}\left(K_{n}\right)=n-1$.

\subsection{The Weisfeiler-Leman Algorithm}

The $k$-dimensional Weisfeiler-Leman algorithm is a procedure that, given a graph $G$ and a colouring of the $k$-tuples of the vertices, computes an isomorphism-invariant refinement of the colouring. Let $\chi_{1}, \chi_{2}: V^{k} \rightarrow \mathcal{C}$ be colourings of the $k$-tuples of vertices of $G$, where $\mathcal{C}$ is some finite set of colours. We say $\chi_{1}$ refines $\chi_{2}\left(\chi_{1} \preceq \chi_{2}\right)$ if for all $\bar{v}, \bar{w} \in V^{k}$ we have

$$
\chi_{1}(\bar{v})=\chi_{1}(\bar{w}) \Rightarrow \chi_{2}(\bar{v})=\chi_{2}(\bar{w}) .
$$

For an integer $k>1$ and a vertex-coloured graph $(G, \chi)$, we first set $\chi_{0}^{G, k}: V^{k} \rightarrow \mathcal{C}$ to be the colouring where each $k$-tuple is coloured by the isomorphism-type of its underlying ordered subgraph. More precisely, $\chi_{0}^{G, k}\left(v_{1}, \ldots, v_{k}\right)=\chi_{0}^{G, k}\left(w_{1}, \ldots, w_{k}\right)$ if and only if for all $i \in[k]$ it holds that $\chi\left(v_{i}\right)=\chi\left(w_{i}\right)$ and for all $i, j \in[k]$ it holds $v_{i}=v_{j} \Leftrightarrow w_{i}=w_{j}$ and $v_{i} v_{j} \in E(G) \Leftrightarrow$ $w_{i} w_{j} \in E(G)$. Then, we recursively define the colouring $\chi_{i+1}^{G, k}$ by setting $\chi_{i+1}^{G, k}\left(v_{1}, \ldots, v_{k}\right):=$ $\left(\chi_{i}^{G, k}\left(v_{1}, \ldots, v_{k}\right) ; \mathcal{M}\right)$, where $\mathcal{M}$ is a multiset defined as

$$
\left\{\left\{\left(\chi_{i}^{G, k}(\bar{v}[w / 1]), \chi_{i}^{G, k}(\bar{v}[w / 2]), \ldots, \chi_{i}^{G, k}(\bar{v}[w / k])\right) \mid w \in V\right\}\right\}
$$

where $\bar{v}[w / i]:=\left(v_{1}, \ldots, v_{i-1}, w, v_{i+1}, \ldots, v_{k}\right)$.

For $k=1$ the definition is similar but we iterate only over the neighbours of $v_{1}$, that is the multiset is defined by $\mathcal{M}:=\left\{\left\{\chi_{i}^{G, 1}(w) \mid w \in N\left(v_{1}\right)\right\}\right.$. The initial colouring $\chi_{0}^{G, 1}$ is simply equal to $\chi$, the vertex-colouring of the input graph.

By definition, every colouring $\chi_{i+1}^{G, k}$ induces a refinement of the partition of the $k$-tuples of the graph $G$ with colouring $\chi_{i}^{G, k}$. Thus, there is some minimal $i$ such that the partition induced by the colouring $\chi_{i+1}^{G, k}$ is not strictly finer than the one induced by the colouring $\chi_{i}^{G, k}$ on $G$. For this minimal $i$, we call the colouring $\chi_{i}^{G, k}$ the stable colouring of $G$ and denote it by $\chi_{(\infty)}^{G, k}$.

For $k=1$ we will usually omit the index $k$ and write $\chi_{(\infty)}^{G}$ instead of $\chi_{(\infty)}^{G, k}$. Also, in some cases we will omit the graph $G$ if it is apparent from context and just write $\chi_{(\infty)}$.

For $k \in \mathbb{N}$, the $k$-dimensional Weisfeiler-Leman algorithm takes as input a coloured graph $(G, \chi)$ and returns the coloured graph $\left(G, \chi_{(\infty)}^{G, k}\right)$. This can be implemented in time $O\left(n^{k+1} \log n\right)$ 
[31]. For two graphs $G$ and $H$, we say that the $k$-dimensional Weisfeiler-Leman algorithm distinguishes $G$ and $H$ if there is some colour $c$ such that the sets $\left\{\bar{v} \mid \bar{v} \in V^{k}(G), \chi_{(\infty)}^{G, k}(\bar{v})=\right.$ $c\}$ and $\left\{\bar{w} \mid \bar{w} \in V^{k}(H), \chi_{(\infty)}^{H, k}(\bar{w})=c\right\}$ have different cardinalities. We write $G \simeq_{k} H$ if the $k$-dimensional Weisfeiler-Leman algorithm does not distinguish between $G$ and $H$. The $k$ dimensional Weisfeiler-Leman algorithm identifies a graph $G$ if it distinguishes $G$ from every non-isomorphic graph $H$.

Pebble Games We will not require details about the information computed by the WeisfeilerLeman algorithm and rather use the following pebble game that is known to capture the same information. Let $k \in \mathbb{N}$. For graphs $G, H$ on the same number of vertices and with vertex colourings $\chi_{G}$ and $\chi_{H}$, respectively, we define the bijective $k$-pebble game $\mathrm{BP}_{k}(G, H)$ as follows:

- The game has two players called Spoiler and Duplicator.

- The game proceeds in rounds. Each round is associated with a pair of positions $(\bar{v}, \bar{w})$ with $\bar{v} \in V(G)^{\ell}$ and $\bar{w} \in V(H)^{\ell}$ where $0 \leq \ell \leq k$.

- The initial position of the game is $((),())$ (the pair of empty tuples).

- Each round consists of the following steps. Suppose the current position of the game is $(\bar{v}, \bar{w})=\left(\left(v_{1}, \ldots, v_{\ell}\right),\left(w_{1}, \ldots, w_{\ell}\right)\right)$. First, Spoiler chooses whether to remove a pair of pebbles or to play a new pair of pebbles. The first option is only possible if $\ell>0$ and the latter option is only possible if $\ell<k$.

If Spoiler wishes to remove a pair of pebbles he picks some $i \in[\ell]$ and the game moves to position $(\bar{v} \backslash i, \bar{w} \backslash i)$ where $\bar{v} \backslash i:=\left(v_{1}, \ldots, v_{i-1}, v_{i+1}, \ldots, v_{\ell}\right)(\bar{w} \backslash i$ is defined in the same way). Otherwise the following steps are performed.

(D) Duplicator picks a bijection $f: V(G) \rightarrow V(H)$.

(S) Spoiler chooses $v \in V(G)$ and sets $w:=f(v)$.

The new position is then $\left(\left(v_{1}, \ldots, v_{\ell}, v\right),\left(w_{1}, \ldots, w_{\ell}, w\right)\right)$.

Spoiler wins the play if for the current position $\left(\left(v_{1}, \ldots, v_{\ell}\right),\left(w_{1}, \ldots, w_{\ell}\right)\right)$ the induced graphs are not isomorphic. More precisely, Spoiler wins if there is an $i \in[\ell]$ such that $\chi_{G}\left(v_{i}\right) \neq \chi_{H}\left(w_{i}\right)$ or there are $i, j \in[\ell]$ such that $v_{i}=v_{j} \nLeftarrow w_{i}=w_{j}$ or $v_{i} v_{j} \in E(G) \nLeftarrow$ $w_{i} w_{j} \in E(H)$. If the play never ends Duplicator wins.

We say that Spoiler (resp. Duplicator) wins the bijective $k$-pebble game $\mathrm{BP}_{k}(G, H)$ if Spoiler (resp. Duplicator) has a winning strategy for the game.

Theorem 2.3 ([5, 28]). Let $G, H$ be two graphs. Then $G \simeq_{k} H$ if and only if Duplicator wins the pebble game $\mathrm{BP}_{k+1}(G, H)$.

Logic There is also a close connection between the Weisfeiler-Leman algorithm and the $k$ variable fragment of first-order logic with counting quantifiers.

As usual first-order logic (FO) is build inductively starting from the atomic formulas. The atomic formulas are of the form $x=y$ and $E x y$ (for this description we restrict the vocabulary to $\{E\}$ where $E$ is a 2-ary relation that corresponds to the edge relation of a graph). Firstorder formulas are build from the atomic formulas in an inductive way using Boolean operations $\wedge, \vee, \neg$, existential quantifiers $\exists x \varphi(x)$ and universal quantifiers $\forall x \varphi(x)$.

We define $C$ to be the extension of FO by counting quantifiers of the form $\exists^{\geq i} x \varphi(x)$. Such a formula is satisfied if there are at least $i$ distinct vertices $v$ that satisfy the formula $\varphi(x)$. Moreover, for $k \in \mathbb{N}$, we let $\mathrm{L}^{k}$ be the $k$-variable fragment of $\mathrm{FO}$, that is those formulas having at most $k$ distinct variables, and similarly we let $\mathrm{C}^{k}$ be the $k$-variable fragment of $\mathrm{C}$. 
Note that while FO and $C$ have the same expressive power this is not true for $\mathrm{L}^{k}$ and $\mathrm{C}^{k}$.

A sentence is a formula without free variables. We say two graphs $G$ and $H$ are equivalent with respect to $C^{k}$, denoted by $G \equiv{ }_{C^{k}} H$, if for every sentence $\varphi$ in the logic $C^{k}$ it holds that $G \models \varphi$ if and only if $H=\varphi$.

With this definition we get the following connection between first-order logic with counting quantifiers and the Weisfeiler-Leman algorithm.

Theorem 2.4 ([5, 28, 31]). Let $G, H$ be two graphs. Then $G \simeq_{k} H$ if and only if $G \equiv_{C^{k+1}} H$.

Corollary 2.5. Let $G$ be a graph that is identified by the $k$-dimensional Weisfeiler-Leman algorithm. Then there is a sentence $\varphi_{G}$ of the logic $C^{k+1}$ that characterises $G$ up to isomorphism.

Proof. Let $n:=|V(G)|$. For every $n$-vertex graph $H$ such that $G ¥ H$ there is a sentence $\psi_{H} \in \mathrm{C}^{k+1}$ over variables $x_{1}, \ldots, x_{k+1}$ such that $G \models \psi_{H}$ and $H \not \models \psi_{H}$. We define

$$
\varphi_{G}:=\exists^{\geq n} x_{1}\left(x_{1}=x_{1}\right) \wedge \neg \exists^{\geq n+1} x_{1}\left(x_{1}=x_{1}\right) \wedge \bigwedge_{H:|V(H)|=n, G \nsucceq H} \psi_{H} .
$$

\subsection{Canonisation}

A common approach to tackle the isomorphism problem is to canonise the input graphs, that is, to compute a standard representation of the input graph that only depends on the isomorphism type of the graph and not on its representation. Formally, a graph canonisation can be defined as follows.

Definition 2.6. A graph canonisation for a graph class $\mathcal{C}$ is a function $\kappa: \mathcal{C} \rightarrow \mathcal{C}$ such that

(1) $\kappa(G) \cong G$ for all $G \in \mathcal{C}$, and

(2) $\kappa(G)=\kappa(H)$ for all graphs $G, H \in \mathcal{C}$ such that $G \cong H$.

Note that the isomorphism problem for a class $\mathcal{C}$ easily reduces to computing a graph canonisation for $\mathcal{C}$. A reduction in the other direction is not known, that is no polynomial-time algorithm is known that reduces the graph canonisation problem for a class $\mathcal{C}$ to the corresponding isomorphism problem. However, most algorithms for the isomorphism problem that are based on combinatorial approaches can be easily turned into graph canonisation algorithms. For example, this is true for isomorphism tests that are based on the Weisfeiler-Leman algorithm.

Theorem 2.7. Let $\mathcal{C}$ be a graph class and suppose the $k$-dimensional Weisfeiler-Leman algorithm identifies all coloured graphs in $\mathcal{C}$. Then there is a graph canonisation for $\mathcal{C}$ that can be computed in time $O\left(n^{k+3} \log n\right)$.

Here, it is assumed that $\mathcal{C}$ is collection of uncoloured graphs (that is closed under isomorphism), and a coloured graph is contained in $C$ if its uncoloured version is in $\mathcal{C}$. This theorem is essentially known among people working on the Weisfeiler-Leman algorithm. Nonetheless we give a proof in Appendix A.

\section{Split Pairs and Flip Functions}

We first show that the $\ell$-dimensional Weisfeiler-Leman algorithm identifies all graphs of rank width at most $k$ for some $\ell \in O(k)$. Let $G$ be a graph of rank width $k$. On a high level, our approach is similar to the proof of the same result for graphs of bounded tree width [21]. For a set $X \subseteq V(G)$ such that $\rho_{G}(X) \leq k$ we wish to find a small set of vertices such that pebbling these vertices splits the graph into multiple sets $C$ that can be treated independently. Moreover, each of these sets $C$ should satisfy that $C \subseteq X$ or $C \subseteq \bar{X}$. As there may be many edges between 
$X$ and $\bar{X}$, it is not obvious how to achieve this. In particular, we cannot simply remove a few vertices in order to separate $X$ from $\bar{X}$. Split pairs and flip functions are our way of dealing with this.

Let $G$ be a graph and $X \subseteq V(G)$. For $v, w \in X$ we define $v \approx_{X} w$ if $N(v) \cap \bar{X}=N(w) \cap \bar{X}$. For $v \in X$ we define the vector $\operatorname{vec}_{X}(v):=\left(a_{v, w}\right)_{w \in \bar{X}} \in \mathbb{F}_{2}^{\bar{X}}$ where $a_{v, w}=1$ if and only if $v w \in E(G)$. Note that $v \approx_{X} w$ if and only if $\operatorname{vec}_{X}(v)=\operatorname{vec}_{X}(w)$. Moreover, for $S \subseteq X$ we define $\operatorname{vec}_{X}(S):=\left\{\operatorname{vec}_{X}(v) \mid v \in S\right\}$.

Lemma 3.1. Let $Y \subseteq X \subseteq V(G)$ and suppose $S \subseteq X$ such that $\operatorname{vec}_{X}(S)$ is linearly independent. Then $\operatorname{vec}_{Y}(S \cap Y)$ is linearly independent.

Proof. We have $\operatorname{vec}_{X}(S \cap Y) \subseteq \operatorname{vec}_{X}(S)$ and thus, $\operatorname{vec}_{X}(S \cap Y)$ is linearly independent. Moreover, $\bar{X} \subseteq \bar{Y}$ which means that every vector $\operatorname{vec}_{Y}(v) \in \operatorname{vec}_{Y}(S \cap Y)$ is an extension of $\operatorname{vec}_{X}(v) \in$ $\operatorname{vec}_{X}(S \cap Y)$. So $\operatorname{vec}_{Y}(S \cap Y)$ is also linearly independent.

For any set of vectors $S \subseteq \mathbb{F}_{2}^{n}$ we denote by $\langle S\rangle$ the linear space spanned by $S$. A set $B \subseteq \mathbb{F}_{2}^{n}$ is a linear basis for $\langle S\rangle$ if $B$ is linearly independent and $\langle B\rangle=\langle S\rangle$.

Definition 3.2. Let $G$ be a graph and $X \subseteq V(G)$. A pair $(A, B)$ is a split pair for $X$ if

(1) $A \subseteq X$ and $B \subseteq \bar{X}$,

(2) $\operatorname{vec}_{X}(A)$ forms a linear basis for $\left\langle\operatorname{vec}_{X}(X)\right\rangle$, and

(3) $\operatorname{vec}_{\bar{X}}(B)$ forms a linear basis for $\left\langle\operatorname{vec}_{\bar{X}}(\bar{X})\right\rangle$.

Note that $|A|=\rho_{G}(X)=\rho_{G}(\bar{X})=|B|$. Also observe that if $(A, B)$ is a split pair for $X$ then $(B, A)$ is a split pair for $\bar{X}$. As a special case the pair $(\emptyset, \emptyset)$ is defined to be a split pair for $X=V(G)$. An ordered split pair for $X$ is a pair $(\bar{a}, \bar{b})=\left(\left(a_{1}, \ldots, a_{q}\right),\left(b_{1}, \ldots, b_{p}\right)\right)$ such that $\left(\left\{a_{1}, \ldots, a_{q}\right\},\left\{b_{1}, \ldots, b_{p}\right\}\right)$ is a split pair for $X$.

Lemma 3.3. Let $G$ be a graph, $X \subseteq V(G)$ and suppose $(A, B)$ is a split pair for $X$. Also let $v, w \in X$ such that $N(v) \cap B=N(w) \cap B$. Then $v \approx_{X} w$. Similarly, $v^{\prime} \approx_{\bar{X}} w^{\prime}$ for all $v^{\prime}, w^{\prime} \in \bar{X}$ such that $N\left(v^{\prime}\right) \cap A=N\left(w^{\prime}\right) \cap A$.

Proof. Let $v, w \in X$ and suppose $B=\left\{b_{1}, \ldots, b_{p}\right\}$. Then, for all $i \in[p]$ we have $v b_{i} \in E(G)$ if and only if $w b_{i} \in E(G)$. Thus

$$
\left(\operatorname{vec}_{\bar{X}}\left(b_{i}\right)\right)_{v}=\left(\operatorname{vec}_{\bar{X}}\left(b_{i}\right)\right)_{w},
$$

that is, the $v$-entry of the vector $\operatorname{vec}_{\bar{X}}\left(b_{i}\right)$ coincides with the $w$-entry. $\operatorname{Since} \operatorname{vec}_{\bar{X}}(B)$ forms a linear basis for $\left\langle\operatorname{vec}_{\bar{X}}(\bar{X})\right\rangle$, we conclude that

$$
\left(\operatorname{vec}_{\bar{X}}\left(v^{\prime}\right)\right)_{v}=\left(\operatorname{vec}_{\bar{X}}\left(v^{\prime}\right)\right)_{w}
$$

for all $v^{\prime} \in \bar{X}$. But this means $N(v) \cap \bar{X}=N(w) \cap \bar{X}$ and thus, $v \approx_{X} w$. The second statement is proved analogously.

For a coloured graph $G=(V, E, \chi)$ and a sequence of vertices $\bar{v}=\left(v_{1}, \ldots, v_{\ell}\right) \in V^{\ell}$ we define $\chi^{\bar{v}}$ to be the colouring obtained from $\chi$ after individualising the vertices in $\bar{v}$ by assigning them the position of their last appearance in $\bar{v}$ and shifting all other colours accordingly. More formally,

$$
\chi^{\bar{v}}: V \rightarrow \mathbb{N}: v \mapsto \begin{cases}i & \text { if } v=v_{i} \wedge \forall j>i: v \neq v_{j} \\ \chi(v)+\ell & \text { otherwise }\end{cases}
$$


Moreover, we denote by $\chi_{(\infty)}^{\bar{v}, G}$ the stable colouring obtained from applying the colour refinement algorithm (i.e. the 1-dimensional Weisfeiler-Leman algorithm) to $\left(G, \chi^{\bar{v}}\right)$. As before, we may omit the graph $G$ if it is clear from context and only write $\chi_{(\infty)}^{\bar{v}}$.

Also, to simplify notation, for tuples $\bar{a}=\left(a_{1}, \ldots, a_{k}\right)$ and $\bar{b}=\left(b_{1}, \ldots, b_{\ell}\right)$ we write $(\bar{a}, \bar{b})$ for the tuple $\left(a_{1}, \ldots, a_{k}, b_{1}, \ldots, b_{\ell}\right)$ obtained from concatenating $\bar{a}$ and $\bar{b}$.

Corollary 3.4. Let $G$ be a graph, $X \subseteq V(G)$ and suppose $(\bar{a}, \bar{b})$ is an ordered split pair for $X$. Also let $v, w \in X$ such that $\chi_{(\infty)}^{(\bar{a}, \bar{b})}(v)=\chi_{(\infty)}^{(\bar{a}, \bar{b})}(w)$. Then $v \approx_{X} w$. Similarly, $v^{\prime} \approx_{\bar{X}} w^{\prime}$ for all $v^{\prime}, w^{\prime} \in \bar{X}$ such that $\chi_{(\infty)}^{(\bar{a}, \bar{b})}\left(v^{\prime}\right)=\chi_{(\infty)}^{(\bar{a}, \bar{b})}\left(w^{\prime}\right)$.

We need to argue how to actually split the graph into independent parts using split pairs. Similar to the previous corollary, we individualise a split pair and perform the colour refinement algorithm. We claim that this graph consists of independent parts as desired. In order to make these parts visible we consider the concept of a flip function.

Definition 3.5. Let $G=(V, E, \chi)$ be a vertex-coloured graph where $\chi: V \rightarrow \mathcal{C}$. A flip function for $G$ is a mapping $f: \mathcal{C} \times \mathcal{C} \rightarrow\{0,1\}$ such that $f\left(c, c^{\prime}\right)=f\left(c^{\prime}, c\right)$ for all $c, c^{\prime} \in \mathcal{C}$.

Moreover, for a graph $G=(V, E, \chi)$ and a flip function $f$ we define the flipped graph $G^{f}=$ $\left(V, E^{f}, \chi\right)$ where

$$
\begin{aligned}
E^{f}:=\quad & \{v w \mid v w \in E \wedge f(\chi(v), \chi(w))=0\} \\
\cup & \{v w \mid v \neq w \wedge v w \notin E \wedge f(\chi(v), \chi(w))=1\} .
\end{aligned}
$$

For a coloured graph $G$ and a flip function $f$ we let $\operatorname{Comp}(G, f) \subseteq 2^{V(G)}$ be the set of vertex sets of the connected components of $G^{f}$. Observe that $\operatorname{Comp}(G, f)$ forms a partition of the vertex set of $G$.

Lemma 3.6. Let $G=(V, E, \chi)$ be a coloured graph and $X \subseteq V(G)$. Also let $(\bar{a}, \bar{b})$ be an ordered split pair for $X$.

Then there is a flip function $f$ for the graph $G^{\prime}=\left(V, E, \chi_{(\infty)}^{(\bar{a}, \bar{b}), G}\right)$ such that for every $C \in$ $\operatorname{Comp}\left(G^{\prime}, f\right)$ it holds that $C \subseteq X$ or $C \subseteq \bar{X}$.

Before diving into the proof, let us briefly discuss the high-level idea. Consider two colour classes $\widehat{P}=\left(\chi_{(\infty)}^{(\bar{a}, \bar{b}), G}\right)^{-1}(c)$ and $\widehat{Q}=\left(\chi_{(\infty)}^{(\bar{a}, \bar{b}), G}\right)^{-1}\left(c^{\prime}\right)$ (for two colours $c$ and $\left.c^{\prime}\right)$. Corollary 3.4 implies that the bipartite graph between $\widehat{P} \cap X$ and $\widehat{Q} \cap \bar{X}$ is either empty or complete. In the latter case, we can simply set $f\left(c, c^{\prime}\right)=1$ to remove all edges between $\widehat{P} \cap X$ and $\widehat{Q} \cap \bar{X}$ in the flipped graph. Now, we only have to ensure that the bipartite graph induced by $\widehat{Q} \cap X$ and $\widehat{P} \cap \bar{X}$ is complete as well (since edges between these two sets are also flipped). However, this follows from the fact that $\chi_{(\infty)}^{(\bar{a}, \bar{b}), G}$ is stable with respect to the colour refinement algorithm.

Proof. Let $\bar{u}:=(\bar{a}, \bar{b})$. We define the flip function $f$ such that $f\left(c, c^{\prime}\right)=1$ if there are $v \in X$ and $w \in \bar{X}$ such that $v w \in E(G)$ and $\left\{\chi_{(\infty)}^{\bar{u}}(v), \chi_{(\infty)}^{\bar{u}}(w)\right\}=\left\{c, c^{\prime}\right\}$. We argue that there are no $v \in X$ and $w \in \bar{X}$ such that $v w$ is an edge in the flipped graph $\left(G^{\prime}\right)^{f}$.

Suppose towards a contradiction this statement does not hold, that is, there are $v \in X$ and $w \in \bar{X}$ such that $v w \in E\left(G^{f}\right)$. Let $c=\chi_{(\infty)}^{\bar{u}}(v)$ and $c^{\prime}=\chi_{(\infty)}^{\bar{u}}(w)$. Then $v w \notin E(G)$, because if $v w \in E(G)$ then $f\left(c, c^{\prime}\right)=1$ and thus $v w \notin E\left(G^{f}\right)$. Moreover, $f\left(c, c^{\prime}\right)=1$, because $v w \notin E(G)$ and $v w \in E\left(G^{f}\right)$. This means that there are $v^{\prime} \in X$ and $w^{\prime} \in \bar{X}$ such that $v^{\prime} w^{\prime} \in E(G)$ and $\left\{\chi_{(\infty)}^{\bar{u}}\left(v^{\prime}\right), \chi_{(\infty)}^{\bar{u}}\left(w^{\prime}\right)\right\}=\left\{c, c^{\prime}\right\}$.

Now we distinguish two cases. The first is that $\chi_{(\infty)}^{\bar{u}}\left(v^{\prime}\right)=c$ and hence, $\chi_{(\infty)}^{\bar{u}}\left(w^{\prime}\right)=c^{\prime}$. Then $v \approx_{X} v^{\prime}$ and $w \approx_{\bar{X}} w^{\prime}$ by Corollary 3.4. But this implies that

$$
v w \in E(G) \Leftrightarrow v w^{\prime} \in E(G) \Leftrightarrow v^{\prime} w^{\prime} \in E(G)
$$



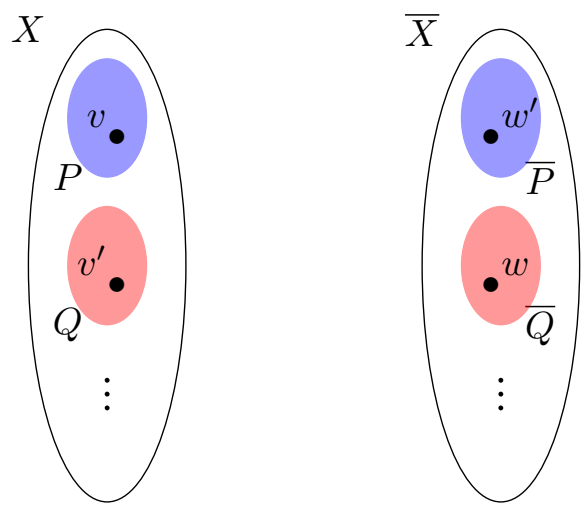

Figure 1: Visualisation of the sets $P, \bar{P}, Q$ and $\bar{Q}$ from the proof of Lemma 3.6.

which is a contradiction.

Let us turn to the second, more complicated, case that $\chi_{(\infty)}^{\bar{u}}\left(v^{\prime}\right)=c^{\prime}$ and $\chi_{(\infty)}^{\bar{u}}\left(w^{\prime}\right)=c$. Let $P=\left(\chi_{(\infty)}^{\bar{u}}\right)^{-1}(c) \cap X, \bar{P}=\left(\chi_{(\infty)}^{\bar{u}}\right)^{-1}(c) \cap \bar{X}, Q=\left(\chi_{(\infty)}^{\bar{u}}\right)^{-1}\left(c^{\prime}\right) \cap X$ and $\bar{Q}=\left(\chi_{(\infty)}^{\bar{u}}\right)^{-1}\left(c^{\prime}\right) \cap \bar{X}$. So $v \in P, v^{\prime} \in Q, w \in \bar{Q}$ and $w^{\prime} \in \bar{P}$ (see Figure 1 ).

Claim 3.7. Let $y \in P$ and $z \in \bar{Q}$. Then $y z \notin E(G)$.

Proof. We have $v \approx_{X} y$ and $w \approx_{\bar{X}} z$ by Corollary 3.4. Hence,

$$
v w \in E(G) \Leftrightarrow v z \in E(G) \Leftrightarrow y z \in E(G) .
$$

Claim 3.8. Let $y \in Q$ and $z \in \bar{P}$. Then $y z \in E(G)$.

Proof. We have $v^{\prime} \approx_{X} y$ and $w^{\prime} \approx_{\bar{X}} z$ by Corollary 3.4. Hence,

$$
v^{\prime} w^{\prime} \in E(G) \Leftrightarrow v^{\prime} z \in E(G) \Leftrightarrow y z \in E(G) .
$$

Now $|N(v) \cap Q|=|N(v) \cap(Q \cup \bar{Q})|=\left|N\left(w^{\prime}\right) \cap(Q \cup \bar{Q})\right| \geq|Q|$ by Claim 3.7 and 3.8. This means $Q \subseteq N(v)$. In particular, $v \in N\left(v^{\prime}\right)$. It follows from Claim 3.8 that $\bar{P} \subseteq N\left(v^{\prime}\right)$. Thus $\left|N\left(v^{\prime}\right) \cap(P \cup \bar{P})\right| \geq|\bar{P}|+1$. Since $\chi_{(\infty)}^{\bar{u}}\left(v^{\prime}\right)=\chi_{(\infty)}^{\bar{u}}(w)=c^{\prime}$ we conclude that $|N(w) \cap(P \cup \bar{P})| \geq$ $|\bar{P}|+1$. But $|N(w) \cap(P \cup \bar{P})|=|N(w) \cap \bar{P}| \leq|\bar{P}|$ by Claim 3.7. This is a contradiction.

To be able to treat the connected components of the flipped graph independently we need to argue that applying a flip function to two graphs neither changes the isomorphism problem nor the effect of the Weisfeiler-Leman algorithm.

Lemma 3.9. Let $G, G^{\prime}$ be two coloured graphs and let $f$ be a flip function for $G$ and $G^{\prime}$. Also let $\varphi: V(G) \rightarrow V\left(G^{\prime}\right)$ be a bijection. Then $\varphi: G \cong G^{\prime}$ if and only if $\varphi: G^{f} \cong\left(G^{\prime}\right)^{f}$.

Proof. Trivial.

Lemma 3.10. Let $G=(V, E, \chi), G^{\prime}=\left(V^{\prime}, E^{\prime}, \chi^{\prime}\right)$ be two coloured graphs and let $f$ be a flip function for $G$ and $G^{\prime}$. Also let $(\bar{v}, \bar{w})=\left(\left(v_{1}, \ldots, v_{k}\right),\left(w_{1}, \ldots, w_{k}\right)\right)$ be a position in the $k$ bijective pebble game $\mathrm{BP}_{k}\left(G, G^{\prime}\right)$. Then Spoiler wins from $(\bar{v}, \bar{w})$ in $\mathrm{BP}_{k}\left(G, G^{\prime}\right)$ if and only if Spoiler wins from $(\bar{v}, \bar{w})$ in $\mathrm{BP}_{k}\left(G^{f},\left(G^{\prime}\right)^{f}\right)$.

Proof. A position $(\bar{v}, \bar{w})$ in the pebble game $\mathrm{BP}_{k}\left(G, G^{\prime}\right)$ is a winning position for Spoiler (i.e., the ordered subgraphs induced by $\bar{v}$ and $\bar{w}$ are not isomorphic) if and only if it is a winning position for Spoiler in the game $\mathrm{BP}_{k}\left(G^{f},\left(G^{\prime}\right)^{f}\right)$.

For two colourings $\chi, \chi^{\prime}: V \rightarrow \mathcal{C}$ we write $\chi \equiv \chi^{\prime}$ if $\chi \preceq \chi^{\prime}$ and $\chi^{\prime} \preceq \chi$, that is the partitions induced by the colour classes are the same for both colourings. 
Corollary 3.11. Let $G=(V, E, \chi)$ be a coloured graph and let $f$ be a flip function for $G$. Then $\chi_{(\infty)}^{G} \equiv \chi_{(\infty)}^{G, f}$ where $\chi_{(\infty)}^{G, f}$ is the stable colouring computed by colour refinement applied to the graph $G^{f}$.

Proof. It holds that $\chi_{(\infty)}^{G}(v)=\chi_{(\infty)}^{G}(w)$ if and only if Spoiler wins from position $((v, v),(w, w))$ in the game $\mathrm{BP}_{2}(G, G)[5,28,31]$. So the statement follows from Lemma 3.10.

For $\bar{v}=\left(v_{1}, \ldots, v_{k}\right) \in V^{k}$ and $C \subseteq V$ we define the tuple $\bar{v} \cap C=\left(v_{i}\right)_{i \in I}$ where $I=$ $\left\{i \in[k] \mid v_{i} \in C\right\}$. Also, for a second tuple $\bar{w}=\left(w_{1}, \ldots, w_{\ell}\right) \in V^{\ell}$, we write $\bar{v} \subseteq \bar{w}$ if $\left\{v_{1}, \ldots, v_{k}\right\} \subseteq\left\{w_{1}, \ldots, w_{\ell}\right\}$.

Corollary 3.12. Let $G=(V, E, \chi), G^{\prime}=\left(V^{\prime}, E^{\prime}, \chi^{\prime}\right)$ be two coloured graphs and let $f$ be a flip function for $G$ and $G^{\prime}$. Let $\bar{v} \in V^{k}$ and $\bar{v}^{\prime} \in\left(V^{\prime}\right)^{k}$. Let $C$ be a connected component of $G^{f}$ such that $\chi(u) \neq \chi(w)$ for all $u \in C, w \in V \backslash C$, and let $C^{\prime}$ a connected component of $\left(G^{\prime}\right)^{f}$ such that $\chi^{\prime}\left(u^{\prime}\right) \neq \chi^{\prime}\left(w^{\prime}\right)$ for all $u^{\prime} \in C^{\prime}, w^{\prime} \in V^{\prime} \backslash C^{\prime}$. Suppose that

$$
\left(G[C], \chi_{(\infty)}^{\bar{v}, G}\right) \nRightarrow\left(G^{\prime}\left[C^{\prime}\right], \chi_{(\infty)}^{\bar{v}^{\prime}, G^{\prime}}\right) .
$$

Let $\bar{w}=\bar{v} \cap C$ and $\bar{w}^{\prime}=\bar{v}^{\prime} \cap C^{\prime}$. Then

$$
\left(G[C], \chi_{(\infty)}^{\bar{w}, G}\right) \nRightarrow\left(G^{\prime}\left[C^{\prime}\right], \chi_{(\infty)}^{\bar{w}^{\prime}, G^{\prime}}\right)
$$

or $\left(G, \chi^{\bar{v}}\right) \varkappa_{1}\left(G^{\prime},\left(\chi^{\prime}\right)^{\bar{v}^{\prime}}\right)$.

Proof. Suppose $\bar{v}=\left(v_{1}, \ldots, v_{k}\right)$ and $\bar{v}^{\prime}=\left(v_{1}^{\prime}, \ldots, v_{k}^{\prime}\right)$. Let $I:=\left\{i \in[k] \mid v_{i} \in C\right\}$ and $I^{\prime}:=\left\{i \in[k] \mid v_{i}^{\prime} \in C^{\prime}\right\}$. Suppose that $\left(G, \chi^{\bar{v}}\right) \simeq_{1}\left(G^{\prime},\left(\chi^{\prime}\right)^{\bar{v}^{\prime}}\right)$. Then $\left(G^{f}, \chi^{\bar{v}}\right) \simeq_{1}\left(\left(G^{\prime}\right)^{f},\left(\chi^{\prime}\right)^{\bar{v}^{\prime}}\right)$ by Lemma 3.10 and Theorem 2.3 and thus, $I=I^{\prime}$. Now suppose

$$
\varphi:\left(G[C], \chi_{(\infty)}^{\bar{w}, G}\right) \cong\left(G^{\prime}\left[C^{\prime}\right], \chi_{(\infty)}^{\bar{w}^{\prime}, G^{\prime}}\right) .
$$

Since $I=I^{\prime}$ it follows that

$$
\varphi:\left(G[C], \chi^{\bar{v}}\right) \cong\left(G^{\prime}\left[C^{\prime}\right],\left(\chi^{\prime}\right)^{\bar{v}^{\prime}}\right) .
$$

Now a simple inductive argument gives that

$$
\varphi:\left(G[C], \chi_{i}^{\bar{v}, G}\right) \cong\left(G^{\prime}\left[C^{\prime}\right], \chi_{i}^{\bar{v}^{\prime}, G^{\prime}}\right)
$$

for all $i \in \mathbb{N}$ since colour refinement only takes colours of neighbours into account. Note that there is no difference between performing colour refinement on $G\left(\operatorname{resp} . G^{\prime}\right)$ or $G^{f}\left(\operatorname{resp} .\left(G^{\prime}\right)^{f}\right)$ by Corollary 3.11.

\section{Weisfeiler-Leman for Graphs of Bounded Rank Width}

In this section we give a proof of Theorem 1.1. The basic strategy for the proof is simple. Given two non-isomorphic graphs $G$ and $H$, where $G$ has rank width at most $k$, we give a winning strategy for Spoiler in the game $\operatorname{BP}_{\ell}(G, H)$ for $\ell=3 k+5$. Spoiler's strategy in the game is to play along a rank decomposition $(T, \gamma)$ for the graph $G$. At a specific node $t \in V(T)$ of the rank decomposition, Spoiler plays an ordered split pair $(\bar{a}, \bar{b})$ for the set $\gamma(t)$ and identifies some component $C$ (with respect to some flip function) that is different from the corresponding component (specified by the bijection chosen by Duplicator) in the second graph. In order to distinguish these components, Spoiler continues to play along the rank decomposition going down the tree. A crucial step to realise this strategy is to ensure that we can remove the pebbles from an ordered split pair of $t$ once Spoiler has pebbled ordered split pairs of the children of $t$. Towards this end, we introduce the notion of nice (triples of) split pairs.

For sets $X, X_{1}, X_{2}$ we write $X=X_{1} \uplus X_{2}$ to denote that $X$ is the disjoint union of $X_{1}$ and $X_{2}$, that is, $X=X_{1} \cup X_{2}$ and $X_{1} \cap X_{2}=\emptyset$. 
Definition 4.1. Let $G$ be a graph and $X, X_{1}, X_{2} \subseteq V(G)$ such that $X=X_{1} \uplus X_{2}$. Let $(A, B)$ be a split pair for $X$ and let $\left(A_{i}, B_{i}\right)$ be a split pair for $X_{i}, i \in\{1,2\}$. We say that $\left(A_{i}, B_{i}\right)$, $i \in\{1,2\}$, are nice (with respect to $(A, B)$ ) if

(1) $A \cap X_{i} \subseteq A_{i}$, and

(2) $B_{3-i} \cap X_{i} \subseteq A_{i}$

for both $i \in\{1,2\}$.

Naturally, a triple of ordered split pairs is nice if the underlying unordered triple of split pairs is nice.

Lemma 4.2. Let $G$ be a graph and $X, X_{1}, X_{2} \subseteq V(G)$ such that $X=X_{1} \uplus X_{2}$. Let $(A, B)$ be a split pair for $X$. Then there are nice split pairs $\left(A_{i}, B_{i}\right)$ for $X_{i}, i \in\{1,2\}$, such that additionally $B_{i} \cap \bar{X} \subseteq B$.

Proof. We first pick $A_{i}$ for both $i \in\{1,2\}$. Since $X_{i} \subseteq X$ we can choose $A_{i}$ in such a way that $A \cap X_{i} \subseteq A_{i}$ by Lemma 3.1.

The set $\operatorname{vec}_{X_{3-i}}\left(A_{3-i}\right)$ spans every element in the set $\operatorname{vec}_{X_{3-i}}\left(X_{3-i}\right) \subseteq \mathbb{F}_{2}^{X} \cup \bar{X}$. Hence, $\operatorname{vec}_{\overline{X_{i}}}\left(A_{3-i}\right)$ spans every element in the set $\operatorname{vec}_{\overline{X_{i}}}\left(X_{3-i}\right) \subseteq \mathbb{F}_{2}^{X_{i}}$.

Moreover, the set $\operatorname{vec}_{\bar{X}}(B)$ spans every element in the set $\operatorname{vec}_{\bar{X}}(\bar{X}) \subseteq \mathbb{F}_{2}^{X_{1} \cup X_{2}}$. $\operatorname{So~}_{\operatorname{vec}_{\overline{X_{i}}}}(B)$ spans every element in the set $\operatorname{vec}_{\overline{X_{i}}}(\bar{X}) \subseteq \mathbb{F}_{2}^{X_{i}}$.

Together this means that $\operatorname{vec}_{\overline{X_{i}}}\left(B \cup A_{3-i}\right)$ spans every element in the set $\operatorname{vec}_{\overline{X_{i}}}\left(\overline{X_{i}}\right) \subseteq \mathbb{F}_{2}^{X_{i}}$. We choose $B_{i} \subseteq B \cup A_{3-i}$ inclusionwise maximal such that $\operatorname{vec}_{\overline{X_{i}}}\left(B_{i}\right)$ is linearly independent.

We remark that the additional guarantee $B_{i} \cap \bar{X} \subseteq B$ is not relevant to obtain a linear upper bound for the Weisfeiler-Leman dimension of graphs of rank width at most $k$. However, the additional overlap between the sets allows us to improve on the constant factors appearing in our arguments.

Also, we shall need the following simple observation. Let $G$ be a graph. A component partition of $G$ is a partition $\mathcal{P}$ of $V(G)$ such that every connected component of $G$ is contained in one block of $\mathcal{P}$, i.e., for every connected component $C$ of $G$ there is some $P \in \mathcal{P}$ such that $C \subseteq P$.

Observation 4.3. Let $G, H$ be two non-isomorphic graphs and let $\mathcal{P}, \mathcal{Q}$ be component partitions of $G$ and $H$, respectively. Also let $\sigma: V(G) \rightarrow V(H)$ be any bijection. Then there is some $v \in V(G)$ such that $G[P] \neq H[Q]$ where $P \in \mathcal{P}$ is the unique set such that $v \in P$ and $Q \in \mathcal{Q}$ is the unique set such that $\sigma(v) \in Q$.

Proof. Since $G \nsubseteq H$ there is some graph $F$ such that

$$
|\{P \in \mathcal{P} \mid G[P] \cong F\}|>|\{Q \in \mathcal{Q} \mid H[Q] \cong F\}| .
$$

Let $\mathcal{P}_{F}:=\{P \in \mathcal{P} \mid G[P] \cong F\}$ and $\mathcal{Q}_{F}:=\{Q \in \mathcal{Q} \mid H[Q] \cong F\}$. Also let $\widehat{\mathcal{P}}_{F}:=\bigcup_{P \in \mathcal{P}_{F}} P$ and $\widehat{\mathcal{Q}}_{F}:=\bigcup_{Q \in \mathcal{Q}_{F}} Q$. Then

$$
\left|\widehat{\mathcal{P}}_{F}\right|>\left|\widehat{\mathcal{Q}}_{F}\right| .
$$

So there is some $v \in \widehat{\mathcal{P}}_{F}$ such that $\sigma(v) \notin \widehat{\mathcal{Q}}_{F}$. Hence, $G[P] \not \models H[Q]$ where $P \in \mathcal{P}$ is the unique set such that $v \in P$ and $Q \in \mathcal{Q}$ is the unique set such that $\sigma(v) \in Q$.

Theorem 4.4 (Theorem 1.1 restated). The $(3 k+4)$-dimensional Weisfeiler-Leman algorithm identifies every graph of rank width at most $k$. 
Proof. Let $G=\left(V(G), E(G), \chi_{G}\right)$ be a graph such that $\operatorname{rw}(G) \leq k$ and moreover let $H=$ $\left(V(H), E(H), \chi_{H}\right)$ be a second graph such that $G \neq H$. Let $(T, \gamma)$ be a rank decomposition of width $k$ for the graph $G$.

We argue that Spoiler wins the bijective $\ell$-pebble game played over graphs $G$ and $H$ where $\ell=3 k+5$. In combination with Theorem 2.3 this proves the theorem. Actually, we first give a winning strategy for Spoiler that requires $\ell=6 k+5$ many pebbles. Then we proceed to argue how to realise this strategy using only $3 k+5$ many pebbles.

On a high-level, Spoiler's strategy is to play along the rank decomposition $(T, \gamma)$ and "confine the non-isomorphism" to smaller and smaller parts of $G$ and $H$. More precisely, for a node $t \in V(T)$, Spoiler's idea is to pebble an ordered split pair $(\bar{a}, \bar{b})$ of $X=\gamma(t)$. Let $f$ be the flip function obtained from Lemma 3.6 with respect to $X$. To "confine the non-isomorphism" to a subset of $X$, Spoiler identifies non-isomorphic components $C \subseteq X$ and $C^{\prime} \subseteq V(H)$ in the flipped graphs $G^{f}$ and $H^{f}$ (after individualising a split pair and performing colour refinement). To remember the components $C$ and $C^{\prime}$, Spoiler places additional "component marker" pebbles on some vertices $v \in C$ and $v^{\prime} \in C^{\prime}$. To eventually reach a winning position, Spoiler's idea is to find such components for nodes $t$ which are further and further away from the root of $T$, eventually ending up at a leaf of $T$ at which point $|C|=1$ and Spoiler has a simple winning strategy. In the following, we describe how this high-level strategy can be realized.

For a node $t \in V(T)$ a tuple $(\bar{a}, \bar{b})$ is an ordered split pair for $t$ if $(\bar{a}, \bar{b})$ is an ordered split pair for $\gamma(t)$.

Now suppose the play is at a position $\left((\bar{a}, \bar{b}, v),\left(\bar{a}^{\prime}, \bar{b}^{\prime}, v^{\prime}\right)\right)$ such that the following conditions are satisfied:

- There is a node $t \in V(T)$ such that $(\bar{a}, \bar{b})$ is an ordered split pair for $t$.

- $v \in \gamma(t)$.

- Let $f$ be the flip function obtained from Lemma 3.6 with respect to $X=\gamma(t)$. Let $C \in \operatorname{Comp}\left(\left(G, \chi_{(\infty)}^{(\bar{a}, \bar{b})}\right), f\right)$ such that $v \in C$. Similarly let $C^{\prime} \in \operatorname{Comp}\left(\left(H, \chi_{(\infty)}^{\left(\bar{a}^{\prime}, \bar{b}^{\prime}\right)}\right), f\right)$ such that $v^{\prime} \in C^{\prime}$. Then

$$
\left(G[C], \chi_{(\infty)}^{(\bar{a}, \bar{b}, v)}\right) \nRightarrow\left(H\left[C^{\prime}\right], \chi_{(\infty)}^{\left(\bar{a}^{\prime}, \bar{b}^{\prime}, v^{\prime}\right)}\right) .
$$

Note that $C \subseteq X$ by Lemma 3.6. Also observe that $\chi_{(\infty)}^{(\bar{a}, \bar{b}, v)}\left(u_{1}\right) \neq \chi_{(\infty)}^{(\bar{a}, \bar{b}, v)}\left(u_{2}\right)$ for all $u_{1} \in C$ and $u_{2} \in V(G) \backslash C$. This is clear for the graph $G^{f}$ since $v \in C$ and $C$ forms a connected component in $G^{f}$ and thus, it also holds for $G$ by Corollary 3.11. The same statement holds for the set $C^{\prime}$ in the graph $H$.

Initially it is easy for Spoiler to reach such a position for the root node of $T$ (choosing the empty split pair $((),())$ and using Observation 4.3). Also observe that in a position as described above the number of pebbles is at most $2 k+1$. We now prove by induction on $|\gamma(t)|$ that Spoiler wins from such a position.

For the base step suppose that $|\gamma(t)|=1$. In this case $C=\{v\}$ and Spoiler easily wins using two additional pebbles. Recall that the sets $C$ and $C^{\prime}$ can be recognised by colour refinement since one of the vertices in each set is individualised (cf. Corollary 3.11).

So for the inductive step suppose $|\gamma(t)|>1$. Let $t_{1}$ and $t_{2}$ be the children of $t$. Let $X:=\gamma(t)$ and $X_{i}:=\gamma\left(t_{i}\right)$ for $i \in\{1,2\}$. Note that $X=X_{1} \uplus X_{2}$.

Let $\left(\bar{a}_{i}, \bar{b}_{i}\right), i \in\{1,2\}$, be nice ordered split pairs for $t_{i}$ (cf. Lemma 4.2). Now Spoiler plays pebbles on $\left(\bar{a}_{1}, \bar{b}_{1}, \bar{a}_{2}, \bar{b}_{2}\right)$ and let $\left(\bar{a}_{1}^{\prime}, \bar{b}_{1}^{\prime}, \bar{a}_{2}^{\prime}, \bar{b}_{2}^{\prime}\right)$ be Duplicator's answer. We also define $\bar{\alpha}:=\left(\bar{a}, \bar{b}, \bar{a}_{1}, \bar{b}_{1}, \bar{a}_{2}, \bar{b}_{2}, v\right)$ and $\bar{\alpha}^{\prime}:=\left(\bar{a}^{\prime}, \bar{b}^{\prime}, \bar{a}_{1}^{\prime}, \bar{b}_{1}^{\prime}, \bar{a}_{2}^{\prime}, \bar{b}_{2}^{\prime}, v^{\prime}\right)$. On an intuitive level, the advantage of pebbling nice ordered split pairs is that, for $i \in\{1,2\}$, we can remove the pebbles $(\bar{a}, \bar{b})$ and $\left(\bar{a}_{3-i}, \bar{b}_{3-i}\right)$ without unpebbling some element from $X_{i}$. 


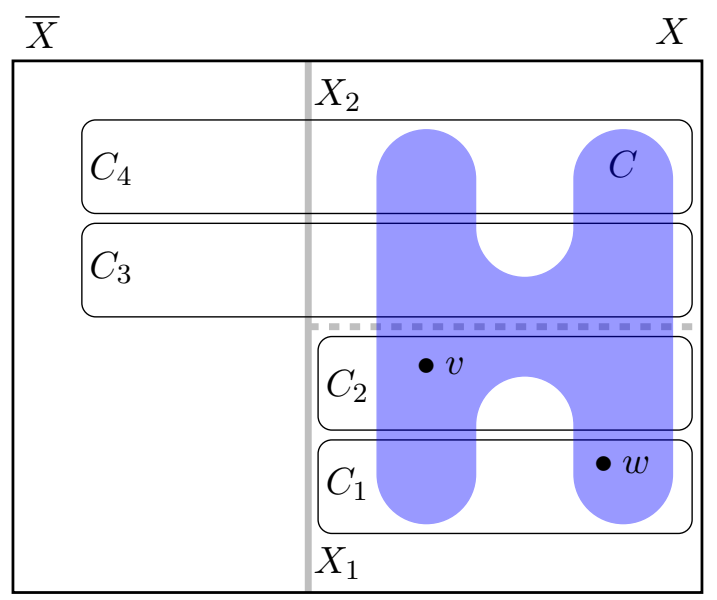

Figure 2: Visualisation for the induction step (Case 1) in the proof of Theorem 4.4.

Let $f_{i}$ be the flip function obtained from Lemma 3.6 with respect to the ordered split pair $\left(\bar{a}_{i}, \bar{b}_{i}\right)$ and the set $X_{i}$. Now Spoiler wishes to play another pebble. Let $\sigma: V(G) \rightarrow V(H)$ be the bijection chosen by Duplicator. Without loss of generality we can assume that

(a) $\sigma(\bar{\alpha})=\bar{\alpha}^{\prime}$, and

(b) $\sigma(C)=C^{\prime}$

(otherwise, Spoiler wins the game using two additional pebbles). Additionally, we can assume without loss of generality that $v \in X_{1}$ (otherwise we swap the roles of $X_{1}$ and $X_{2}$ ).

First consider the flip function $f_{1}$ (see Figure 2). Let $\left\{C_{1}, \ldots, C_{p}\right\}=\left\{D \in \operatorname{Comp}\left(G, f_{1}\right) \mid\right.$ $D \cap C \neq \emptyset\}$. Similarly, let $\left\{C_{1}^{\prime}, \ldots, C_{p^{\prime}}^{\prime}\right\}=\left\{D^{\prime} \in \operatorname{Comp}\left(H, f_{1}\right) \mid D^{\prime} \cap C^{\prime} \neq \emptyset\right\}$. Clearly,

$$
\left(\left(G^{f_{1}}\right)[C], \chi_{(\infty)}^{\bar{\alpha}}\right) \approx\left(\left(H^{f_{1}}\right)\left[C^{\prime}\right], \chi_{(\infty)}^{\bar{\alpha}^{\prime}}\right)
$$

using Lemma 3.9. By Observation 4.3 there is some $w \in C$ such that

$$
\left(\left(G^{f_{1}}\right)\left[C \cap C_{i}\right], \chi_{(\infty)}^{\bar{\alpha}}\right) \nRightarrow\left(\left(H^{f_{1}}\right)\left[C^{\prime} \cap C_{i^{\prime}}^{\prime}\right], \chi_{(\infty)}^{\bar{\alpha}^{\prime}}\right)
$$

where $i \in[p]$ is the unique index such that $w \in C_{i}$ and $i^{\prime} \in\left[p^{\prime}\right]$ is the unique index such that $\sigma(w) \in C_{i^{\prime}}^{\prime}$. Without loss of generality suppose that $i=i^{\prime}=1$. Applying Lemma 3.9 once again, we get that

$$
\left(G\left[C \cap C_{1}\right], \chi_{(\infty)}^{\bar{\alpha}}\right) \approx\left(H\left[C^{\prime} \cap C_{1}^{\prime}\right], \chi_{(\infty)}^{\bar{\alpha}^{\prime}}\right) .
$$

Also, by Lemma 3.6, it holds that $C_{1} \subseteq X_{1}$ or $C_{1} \subseteq \overline{X_{1}}$. In particular, $C \cap C_{1} \subseteq X_{1}$ or $C \cap C_{1} \subseteq X_{2}$.

Case 1: $C \cap C_{1} \subseteq X_{1}$.

Observe that $\chi_{(\infty)}^{\bar{\alpha}}\left(u_{1}\right) \neq \chi_{(\infty)}^{\bar{\alpha}}\left(u_{2}\right)$ for all $u_{1} \in C$ and $u_{2} \in V(G) \backslash C$. Hence, we get that

$$
\left(G\left[C_{1}\right], \chi_{(\infty)}^{\bar{\alpha}}\right) \varsubsetneqq\left(H\left[C_{1}^{\prime}\right], \chi_{(\infty)}^{\bar{\alpha}^{\prime}}\right) .
$$

Now Spoiler plays the next pebble as follows: if $v \in C_{1}$ and $v^{\prime} \in C_{1}^{\prime}$ then he plays $z=v$ and $z^{\prime}=v^{\prime}$, otherwise Spoiler plays $z=w$ and $z^{\prime}=\sigma(w)$. Clearly,

$$
\left(G\left[C_{1}\right], \chi_{(\infty)}^{(\bar{\alpha}, z)}\right) \approx\left(H\left[C_{1}^{\prime}\right], \chi_{(\infty)}^{\left(\bar{\alpha}^{\prime}, z^{\prime}\right)}\right)
$$


Now consider again the flip function $f_{1}$. In $G^{f_{1}}$ the set $C_{1}$ forms a connected component and similarly, in $H^{f_{1}}$ the set $C_{1}^{\prime}$ forms a connected component. Hence, removing any pebbles from vertices outside $C_{1}$ (resp. $C_{1}^{\prime}$ ) does not affect the stable colouring restricted to the component $C_{1}$ (resp. $\left.C_{1}^{\prime}\right)$ by Corollary 3.11. Since all pebbles $\left(\bar{a}, \bar{b}, \bar{a}_{2}, \bar{b}_{2}, v\right)$ (resp. $\left.\left(\bar{a}^{\prime}, \bar{b}^{\prime}, \bar{a}_{2}^{\prime}, \bar{b}_{2}^{\prime}, v^{\prime}\right)\right)$ are either outside of $C_{1}$ or the corresponding vertices are also pebbled using $\left(\bar{a}_{1}, \bar{b}_{1}, z\right)$ (resp. $\left.\left(\bar{a}_{1}^{\prime}, \bar{b}_{1}^{\prime}, z^{\prime}\right)\right)$, we can remove the pebbles $\left(\bar{a}, \bar{b}, \bar{a}_{2}, \bar{b}_{2}, v\right)$ and $\left(\bar{a}^{\prime}, \bar{b}^{\prime}, \bar{a}_{2}^{\prime}, \bar{b}_{2}^{\prime}, v^{\prime}\right)$ and still get that

$$
\left(G\left[C_{1}\right], \chi_{(\infty)}^{\left(\bar{a}_{1}, \bar{b}_{1}, z\right)}\right) \nRightarrow\left(H\left[C_{1}^{\prime}\right], \chi_{(\infty)}^{\left(\bar{a}_{1}^{\prime}, \bar{b}_{1}^{\prime}, z^{\prime}\right)}\right)
$$

by Corollary 3.12 (or Spoiler wins using two additional pebbles). But now we can apply the induction hypothesis to $t_{1}$. As a result, Spoiler wins from the current position and hence, Spoiler wins from position $\left((\bar{a}, \bar{b}, v),\left(\bar{a}^{\prime}, \bar{b}^{\prime}, v^{\prime}\right)\right)$.

Case 2: $C \cap C_{1} \subseteq X_{2}$.

Let us first remark that this case is not symmetric to the first case since the set $C_{1}$ is defined with respect to the flip function $f_{1}$. Also, for ease of notation, define $M:=C \cap C_{1}$ and $M^{\prime}:=C^{\prime} \cap C_{1}^{\prime}$.

First Spoiler plays the next pebble on $w$ and $w^{\prime}:=\sigma(w)$. Observe that $\chi_{(\infty)}^{(\bar{\alpha}, w)}\left(u_{1}\right) \neq$ $\chi_{(\infty)}^{(\bar{\alpha}, w)}\left(u_{2}\right)$ for all $u_{1} \in M$ and $u_{2} \in V(G) \backslash M$.

Now consider the flip function $f_{2}$. Spoiler wishes to play the next pebble. Again, let $\sigma: V(G) \rightarrow V(H)$ denote the bijection chosen by Duplicator. Without loss of generality we can assume that

(a) $\sigma(\bar{\alpha})=\bar{\alpha}^{\prime}$

(b) $\sigma(w)=w^{\prime}$

(c) $\sigma(M)=M^{\prime}$.

(as before, otherwise Spoiler wins the game using two additional pebbles). Consider the flip function $f_{2}$. Let $\left\{D_{1}, \ldots, D_{q}\right\}=\left\{D \in \operatorname{Comp}\left(G, f_{2}\right) \mid D \cap M \neq \emptyset\right\}$. Similarly, let $\left\{D_{1}^{\prime}, \ldots, D_{q^{\prime}}^{\prime}\right\}=\left\{D^{\prime} \in \operatorname{Comp}\left(H, f_{2}\right) \mid D^{\prime} \cap M^{\prime} \neq \emptyset\right\}$. Clearly,

$$
\left(\left(G^{f_{2}}\right)[M], \chi_{(\infty)}^{(\bar{\alpha}, w)}\right) \not\left(\left(H^{f_{2}}\right)\left[M^{\prime}\right], \chi_{(\infty)}^{\left(\bar{\alpha}^{\prime}, w^{\prime}\right)}\right)
$$

using Lemma 3.9. By Observation 4.3 there is some $z \in M$ such that

$$
\left(\left(G^{f_{2}}\right)\left[M \cap D_{i}\right], \chi_{(\infty)}^{(\bar{\alpha}, w)}\right) \not\left(\left(H^{f_{2}}\right)\left[M^{\prime} \cap D_{i^{\prime}}^{\prime}\right], \chi_{(\infty)}^{\left(\bar{\alpha}^{\prime}, w^{\prime}\right)}\right)
$$

where $i \in[q]$ is the unique index such that $z \in D_{i}$ and $i^{\prime} \in\left[q^{\prime}\right]$ is the unique index such that $\sigma(z) \in D_{i^{\prime}}^{\prime}$. As before, we may assume without loss of generality that $i=i^{\prime}=1$. Applying Lemma 3.9 once again, we get that

$$
\left(G\left[M \cap D_{1}\right], \chi_{(\infty)}^{(\bar{\alpha}, w)}\right) \not\left(H\left[M^{\prime} \cap D_{1}^{\prime}\right], \chi_{(\infty)}^{\left(\bar{\alpha}^{\prime}, w^{\prime}\right)}\right) .
$$

Now recall that $\chi_{(\infty)}^{(\bar{\alpha}, w)}\left(u_{1}\right) \neq \chi_{(\infty)}^{(\bar{\alpha}, w)}\left(u_{2}\right)$ for all $u_{1} \in M$ and $u_{2} \in V(G) \backslash M$. This means that

$$
\left(G\left[D_{1}\right], \chi_{(\infty)}^{(\bar{\alpha}, w)}\right) \varsubsetneqq\left(H\left[D_{1}^{\prime}\right], \chi_{(\infty)}^{\left(\bar{\alpha}^{\prime}, w^{\prime}\right)}\right) .
$$

Now Spoiler plays the next pebble as follows: if $w \in D_{1}$ and $w^{\prime} \in D_{1}^{\prime}$ then he plays $x=w$ and $x^{\prime}=w^{\prime}$, otherwise Spoiler plays $x=z$ and $x^{\prime}=\sigma(z)$. Clearly,

$$
\left(G\left[D_{1}\right], \chi_{(\infty)}^{(\bar{\alpha}, w, x)}\right) \nRightarrow\left(H\left[D_{1}^{\prime}\right], \chi_{(\infty)}^{\left(\bar{\alpha}^{\prime}, w^{\prime}, x^{\prime}\right)}\right)
$$


Now consider again the flip function $f_{2}$. In $G^{f_{2}}$ the set $D_{1}$ forms a connected component and similarly, in $H^{f_{2}}$ the set $D_{1}^{\prime}$ forms a connected component. Hence, removing any pebbles from vertices outside $D_{1}$ (resp. $D_{1}^{\prime}$ ) does not affect the stable colouring restricted to the component $D_{1}$ (resp. $\left.D_{1}^{\prime}\right)$ by Corollary 3.11. Since all pebbles $\left(\bar{a}, \bar{b}, \bar{a}_{1}, \bar{b}_{1}, v, w\right)$ (resp. $\left.\left(\bar{a}^{\prime}, \bar{b}^{\prime}, \bar{a}_{1}^{\prime}, \bar{b}_{1}^{\prime}, v^{\prime}, w^{\prime}\right)\right)$ are either outside of $D_{1}$ (recall that $v \in X_{1}$ and hence, $v \notin D_{1}$ ) or the corresponding vertices are also pebbled using $\left(\bar{a}_{2}, \bar{b}_{2}, x\right)$ (resp. $\left(\bar{a}_{2}^{\prime}, \bar{b}_{2}^{\prime}, x^{\prime}\right)$ ), we can remove the pebbles $\left(\bar{a}, \bar{b}, \bar{a}_{1}, \bar{b}_{1}, v, w\right)$ and $\left(\bar{a}^{\prime}, \bar{b}^{\prime}, \bar{a}_{1}^{\prime}, \bar{b}_{1}^{\prime}, v^{\prime}, w^{\prime}\right)$ and still get that

$$
\left(G\left[D_{1}\right], \chi_{(\infty)}^{\left(\bar{a}_{2}, \bar{b}_{2}, x\right)}\right) \varsubsetneqq\left(H\left[D_{1}^{\prime}\right], \chi_{(\infty)}^{\left(\bar{a}_{2}^{\prime}, \bar{b}_{2}^{\prime}, x^{\prime}\right)}\right)
$$

by Corollary 3.12 (or Spoiler wins using two additional pebbles). But now we can apply the induction hypothesis to $t_{2}$. As a result, Spoiler wins from the current position and hence, Spoiler wins from position $\left((\bar{a}, \bar{b}, v),\left(\bar{a}^{\prime}, \bar{b}^{\prime}, v^{\prime}\right)\right)$.

Overall, by the induction principle, this gives us a winning strategy for Spoiler in the pebble game played over the graphs $G$ and $H$. It remains to analyse the number of pebbles required to implement this strategy. Looking at Spoiler's strategy, it is not difficult to see that it requires at most $6 k+5$ many pebbles. More precisely, Spoiler needs $6 k$ pebbles to pebble the three ordered split pairs $(\bar{a}, \bar{b})$ and $\left(\bar{a}_{i}, \bar{b}_{i}\right)$ for $i \in\{1,2\}$. The base step requires three additional pebbles. In the inductive step, five additional pebbles suffice, three for pebbling $v, w$ and $x$ and two pebbles to simulate colour refinement in case the bijections chosen by Duplicator do not match up.

However, taking a closer look, some vertices are always pebbled multiple times due to the nice ordered split pairs. In particular, we get that $\bar{a} \subseteq \bar{a}_{1} \cup \bar{a}_{2}$. Since there is no need to pebble any vertex multiple times, we conclude that Spoiler can also win using only $5 k+5$ many pebbles.

But even this number can be further improved. Indeed, Spoiler can also find nice ordered split pairs $(\bar{a}, \bar{b})$ and $\left(\bar{a}_{i}, \bar{b}_{i}\right)$ for $i \in\{1,2\}$ such that additionally $\bar{b}_{i} \cap \bar{X} \subseteq \bar{b}$ (cf. Lemma 4.2). Then $\bar{b}_{i} \subseteq \bar{b} \cup \bar{a}_{3-i}$ for both $i \in\{1,2\}$. Again, there is no need to pebble any vertex multiple times and hence, Spoiler actually requires only $3 k+5$ many pebbles.

\section{Capturing PTIME on Graphs of Bounded Rank Width}

In this section, we prove Theorem 1.5. We start with a quick introduction to the necessary background from descriptive complexity theory.

\subsection{Preliminaries from Descriptive Complexity Theory}

We assume that the reader has a solid background in logic and, in particular, is familiar with the standard fixed-point logics used in finite model theory. For background and precise definitions, we refer the reader to the textbooks $[12,15,19,30,35]$.

\section{Relational Structures}

We work with finite structures over a relational vocabulary $\tau$. The universe of a structure $A$ is denoted by $V(A)$, and the interpretation of a $k$-ary relation symbol $R$ is denoted by $R(A)$. In particular, we view graphs as structures of vocabulary $\{E\}$ for a binary relation symbol $E$. For a structure $A$ and a subset $U \subseteq V(A)$, the induced substructure $A[U]$ is the structure with universe $V(A[U]):=U$ and relations $R(A[U]):=R(A) \cap U^{k}$ for every $k$-ary relation symbol in the vocabulary. Two structures $A, B$ of the same vocabulary $\tau$ are isomorphic $(A \cong B)$ if there is a bijective mapping, called an isomorphism, $f: V(A) \rightarrow V(B)$ such that for all $k$-ary $R \in \tau$ and all $\bar{a} \in V(A)^{k}$ we have $\bar{a} \in R(A)$ if and only if $f(\bar{a}) \in R(B)$. We extend isomorphisms to structures with individualised elements: for tuples $\bar{v}=\left(v_{1}, \ldots, v_{\ell}\right) \in V(A)^{\ell}$, $\bar{w}=\left(w_{1}, \ldots, w_{\ell}\right) \in V(B)^{\ell}$, an isomorphism from $(A, \bar{v})$ to $(B, \bar{w})$ is an isomorphism $f$ from $A$ to $B$ such that $f\left(v_{i}\right)=w_{i}$ for all $i \in[\ell]$. We write $(A, \bar{v}) \cong(B, \bar{w})$ if such an isomorphism exists. 


\section{Fixed-Point Logic with Counting}

Inflationary fixed-point logic is the extension of first-order logic by a fixed-point operator with an inflationary semantics. Instead of giving a formal definition of its syntax (where we follow [19]) and semantics, we give one illustrative example.

Example 5.1. The IFP-sentence

$$
\text { conn }:=\forall x_{1} \forall x_{2} \text { ifp }\left(X \leftarrow\left(x_{1}, x_{2}\right) \mid x_{1}=x_{2} \vee E\left(x_{1}, x_{2}\right) \vee \exists x_{3}\left(X\left(x_{1}, x_{3}\right) \wedge X\left(x_{3}, x_{2}\right)\right)\right)\left(x_{1}, x_{2}\right)
$$

states that a graph is connected.

IFP-formulas have individual variables, ranging over the elements of the universe of a structure, and relation variables, each with a prescribed arity, ranging over relations of this arity over the universe. We write $\varphi\left(X_{1}, \ldots, X_{k}, x_{1}, \ldots, x_{\ell}\right)$ to denote that the free relation variables of a formula are among $X_{1}, \ldots, X_{k}$ and the free individual variables are among $x_{1}, \ldots, x_{\ell}$. For a structure $A$, relations $R_{1}, \ldots, R_{k}$ of the appropriate arities, and elements $v_{1}, \ldots, v_{\ell}$, we write $A \models \varphi\left(R_{1}, \ldots, R_{k}, v_{1}, \ldots, v_{k}\right)$ to denote that $A$ satisfies $\varphi$ if the $X_{i}$ are interpreted by $R_{i}$ and the $x_{j}$ are interpreted by $v_{j}$.

Inflationary fixed-point logic with counting, $\mathrm{FP}+\mathrm{C}$, is the extension of IFP by counting operators that allow it to speak about cardinalities of definable sets and relations. To define FP+C, we interpret the logic IFP over two-sorted extensions of structures by a numerical sort. For a structure $A$, we let $N(A)$ be the initial segment $\{0, \ldots,|V(A)|\}$ of the nonnegative integers. We let $A^{+}$be the two-sorted structure $A \cup(N(A), \leq)$, where $\leq$ is the natural linear order on $N(A)$. To avoid confusion, we always assume that $V(A)$ and $N(A)$ are disjoint.

In a structure $A$, individual variables of the logic $\mathrm{FP}+\mathrm{C}$ range either over the set $V(A)$ (vertex variables) or over the set $N(A)$ (number variables). Relation variables may range over mixed relations, having certain places for vertices and certain places for numbers. The logic $\mathrm{FP}+\mathrm{C}$ has all the constructors of IFP, and in addition counting terms of the form $\# x \varphi$, where $x$ is a vertex variable and $\varphi$ some formula. The value of this term in a structure $A$ is the number of $v \in V(A)$ such that $A$ satisfies $\varphi$ if $x$ is interpreted by $v$ (under some fixed assignment to the other free variables of $\varphi$ ).

Example 5.2. We start by giving an a FP+C-formula even $(y)$ with one free number variable $y$ stating that $y$ is an even number:

$$
\operatorname{even}(y):=\operatorname{ifp}\left(Y \leftarrow y \mid \forall y^{\prime} y \leq y^{\prime} \vee \exists y^{\prime \prime} \exists y^{\prime}\left(Y\left(y^{\prime}\right) \wedge \operatorname{succ}\left(y^{\prime}, y^{\prime \prime}\right) \wedge \operatorname{succ}\left(y^{\prime \prime}, y\right)\right)\right)(y),
$$

where $\operatorname{succ}\left(z, z^{\prime}\right):=z \leq z^{\prime} \wedge \neg z=z^{\prime} \wedge \forall z^{\prime \prime}\left(z^{\prime \prime} \leq z \vee z^{\prime} \leq z^{\prime \prime}\right)$. Then the following $\mathrm{FP}+\mathrm{C}$-sentence defines the class of Eulerian graphs (that is, graphs with a cyclic walk that traverses all edges, which are well-known to be exactly the connected graphs in which all vertices have even degree):

$$
\text { eulerian := conn } \wedge \forall x \text { even }\left(\# x^{\prime} E\left(x, x^{\prime}\right)\right),
$$

where conn is the sentence from Example 5.1.

We like to think of definitions in the logic $\mathrm{FP}+\mathrm{C}$ in an algorithmic way, where formulas are "programs" computing an input-output relation. Rather than writing out syntactical details, we describe these programs in a high-level form, as we would do with any type of algorithms and leave the "FP+C-implementation" to the reader. A thorough technical treatment of the issues involved in this can be found in [19, Chapters 2 and 3].

We will make assertions about the existence of FP+C-formulas, or "programs", with a specified input-output behaviour. In the most basic setting of an $\mathrm{FP}+\mathrm{C}$-sentence like eulerian, the input is a structure (a graph) and the output a Boolean value. For a formula like even $(y)$ the input 
is a structure $A$ and the output is a subset of $N(A)$. But the input can be more complicated. For example, the input may be a triple $(G, U, d)$, where $G$ is a graph, $U \subseteq V(G), d \in N(G)$, and our task is to write an FP+C-formula that computes the set of all vertices of $G$ that have distance at most $d$ from a vertex in $U$. Formally, this means that we have to write an $\mathrm{FP}+\mathrm{C}-$ formula $\varphi(X, y, x)$ such that for all graphs $G$, all subsets $U \subseteq V(G)$, and all $d \in N(G)$ we have $G \models \varphi(U, d, v)$ if and only if the distance of $v$ to $U$ in $G$ is at most $d$. An even more complicated type of assertion we will frequently see is of the following form: given a tuple $(G, U, v, H)$, where $G$ is a graph, $U \subseteq V(G), v \in V(G) \backslash U$, and $H$ is a graph with vertex set $V(H) \subseteq N(G)$, in $\mathrm{FP}+\mathrm{C}$ we can decide if the connected component of $G \backslash U$ that contains $v$ is isomorphic to $H$. Here our task is to define an $\mathrm{FP}+\mathrm{C}$-formula $\varphi(X, x, Y, Z)$, where $X$ is a unary relation ranging over the vertex sort, $x$ is a vertex variable, and $Y$ and $Z$ are a unary and a binary relation symbol both ranging over the number sort, such that for all graphs $G, U \subseteq V(G), v \in V(G) \backslash U$, $P \subseteq N(G), Q \subseteq N(G)^{2}$ we have $G=\varphi(U, v, P, Q)$ if and only if the connected component of $G \backslash U$ that contains $v$ is isomorphic to the graph $H$ with $V(H)=P$ and $E(H)=Q$. Actually, it is known that such a formula can not exist (see [5]). However, in this work we will only require such formulas for specific graph classes $\mathcal{C}$, that is, when the input is restricted to graphs $G \in \mathcal{C}$.

Lemma 5.3 (cf. [38]). Let $\mathcal{C}$ be a hereditary graph class (i.e., $\mathcal{C}$ is closed under induced subgraphs) such that the $k$-dimensional Weisfeiler-Leman algorithm identifies all graphs $G \in \mathcal{C}$ for some constant number $k$. Then there is an $\mathrm{FP}+\mathrm{C}$-formula $\varphi(X, Y, Z)$, where $X$ is a unary relation ranging over the vertex sort and $Y$ and $Z$ are a unary and a binary relation symbol both ranging over the number sort, such that for all graphs $G \in \mathcal{C}, U \subseteq V(G), P \subseteq N(G), Q \subseteq N(G)^{2}$ we have $G \models \varphi(U, P, Q)$ if any only if $G[U] \cong(P, Q)$.

In a setting where we have several input objects, such as the tuple $(G, U, v, H)$ above, we always have one main input structure, which will be listed first. In the example $(G, U, v, H)$, this is the graph $G$. All other objects are defined relative to this main structure and its numerical extension. In the example, $U$ is a subset of $V(G), v$ an element of $V(G)$, and $H$ a structure with universe $N(G)$. Sometimes, we will have to deal with whole families of structures. They will always be indexed by tuples of elements of the main structure. For example, we may be given a pair $\left(A,\left(H_{(v, p)}\right)_{(v, p) \in V(A) \times N(A)}\right)$ where the $H_{(v, p)}$ are graphs with universe $V\left(H_{(v, p)}\right) \subseteq N(A)$. Formally, we can represent such a family by the ternary relation $R=\{(v, p, q) \in V(A) \times N(A) \times$ $\left.N(A) \mid q \in V\left(H_{(v, p)}\right)\right\}$ and the quaternary relation $S=\left\{\left(v, p, q, q^{\prime}\right) \in V(A) \times N(A) \times N(A) \times\right.$ $\left.N(A) \mid\left(q, q^{\prime}\right) \in E\left(H_{(v, p)}\right)\right\}$.

\section{Definable Canonisation}

Recall from the introduction that a logic captures polynomial time on a class $\mathcal{C}$ of structures if each polynomial-time decidable property of structures in $\mathcal{C}$ is expressible by a sentence of the logic. By the Immerman-Vardi Theorem [29, 44], IFP captures polynomial time on the class of all ordered structures. ${ }^{2}$ A straightforward way of applying this theorem to a class $\mathcal{C}$ of unordered structures is to define a linear order on this class: if there is a formula ord $(x, y)$ of the logic IFP that defines a linear order on all structures in $\mathcal{C}$, then IFP still captures polynomial time on $\mathcal{C}$. Unfortunately, this observation is rarely applicable, because usually it is impossible to define linear orders. For example, it is impossible to define a linear order on a structure that has a nontrivial automorphism.

A much more powerful idea, going back to [31,38] and known as definable canonisation, is to define an ordered copy of the input structure. To implement this idea, FP+C is particularly well-suited, because we can take the numerical part $N(A)$ of a structure $A$ as the universe of the

\footnotetext{
${ }^{2}$ Originally, the Immerman-Vardi Theorem states that least fixed-point logic LFP captures polynomial time on the class of all ordered structures. However, it is known that LFP and IFP have the same expressive power [27, 34].
} 
ordered copy of $A$. Technically, definable canonisation is based on syntactical interpretations (called transductions in [19]). Instead of introducing the unwieldy machinery of syntactical interpretations in full generality, we just focus on a special case that suffices for our purposes. Suppose, we have a structure $A$ of vocabulary $\tau$. To define an ordered copy of $A$, we need a formula $\varphi_{R}\left(y_{1}, \ldots, y_{k}\right)$ with free number variables $y_{i}$ for every $k$-ary $R \in \tau$. A family $\Phi=$ $\left(\varphi_{R}(\bar{y}) \mid R \in \tau\right)$ of such formulas defines a structure $A^{\Phi}$ with universe $V\left(A^{\Phi}\right):=\{0, \ldots,|V(A)|-$ $1\} \subseteq N(A)$ and relations $R\left(A^{\Phi}\right):=\left\{\left(p_{1}, \ldots, p_{k}\right) \in V\left(A^{\Phi}\right)^{k} \mid A=\varphi_{R}\left(p_{1}, \ldots, p_{k}\right)\right\}$. We say that $\Phi$ defines an ordered copy of $A$ if $A^{\Phi} \cong A$. Observe that if $\Phi$ defines an ordered copy of $A$, then this ordered copy is canonical in the sense that for all $B \cong A$ it holds that $B^{\Phi}=A^{\Phi}$, because we have $N(B)=N(A)=\{0, \ldots, n\}$ for $n=|V(A)|=|V(B)|$, and definitions in the logic $\mathrm{FP}+\mathrm{C}$ are isomorphism-invariant. We say that a class $\mathcal{C}$ of $\tau$-structures admits $\mathrm{FP}+\mathrm{C}$-definable canonisation if there is a family $\Phi=\left(\varphi_{R}(\bar{y}) \mid R \in \tau\right)$ of $\mathrm{FP}+\mathrm{C}$-formulas such that for all $A \in \mathcal{C}$ it holds that $A^{\Phi} \cong A$. The following lemma is a direct consequence of the Immerman-Vardi Theorem (for a proof, see [19, Lemma 3.3.8]).

Lemma 5.4. Let $\mathcal{C}$ be a class of $\tau$-structures that admits $\mathrm{FP}+\mathrm{C}$-definable canonisation. Then $\mathrm{FP}+\mathrm{C}$ captures polynomial time on $\mathcal{C}$.

Sometimes, we need to define ordered copies of substructures of a structure. To define an ordered copy of a substructure of a $\tau$-structure we use a family $\Psi$ of formulas that in addition to formulas $\psi_{R}(\bar{y})$ for the relations contains a formula $\psi_{V}(y)$ that specifies the universe of the ordered copy. Given a pair $(A, B)$, where $A$ is a $\tau$-structure and $B \subseteq A$ a substructure, such a family $\Psi$ defines a structure $B^{\prime}$ with universe $V\left(B^{\prime}\right):=\left\{p \in N(A)|A|=\psi_{V}(p)\right\}$ and relations $R\left(B^{\prime}\right):=\left\{\left(p_{1}, \ldots, p_{k}\right) \in V\left(B^{\prime}\right)^{k} \mid A \models \psi_{R}\left(p_{1}, \ldots, p_{k}\right)\right\}$. If $B^{\prime} \cong B$, we say that $\Psi$ defines an ordered copy of $B$ in $A$.

We will also see more complicated assertions such as the following: given a tuple $(G, U, v)$, where $G$ is a graph, $U \subseteq V(G), v \in V(G) \backslash U$, in $\mathrm{FP}+\mathrm{C}$ we can compute an ordered copy of the connected component of $G \backslash U$ that contains $v$. This means that we can construct FP+C-formulas $\psi_{V}(X, x, y)$ and $\psi_{E}\left(X, x, y_{1}, y_{2}\right)$ such that for all $G, U \subseteq V(G)$, and $v \in V(G) \backslash U$, the graph with universe $V^{\prime}:=\left\{p \in N(G) \mid G=\psi_{V}(U, v, p)\right\}$ and edge relation $E^{\prime}:=\left\{\left(p_{1}, p_{2}\right) \in\left(V^{\prime}\right)^{2} \mid\right.$ $\left.G \models \psi_{E}\left(U, v, p_{1}, p_{2}\right)\right\}$ is isomorphic to the connected component of $v$ in $G \backslash U$.

We will routinely have to compare ordered copies of substructures of our input graphs. To do this, we define a lexicographical order on $\tau$-structures whose universe is an initial segment of the nonnegative integers. First, we fix a linear order of the relation symbols in $\tau$. Say, $\tau=\left\{R_{1}, \ldots, R_{\ell}\right\}$ and we order the $R_{i}$ by their indices. Now let $A, B$ be two $\tau$-structures such that $V(A)=\left\{0, \ldots, n_{A}-1\right\}$ and $V(B)=\left\{0, \ldots, n_{B}-1\right\}$. Structure $A$ is lexicographically smaller than or equal to structure $B$ (we write $A \leq_{\text {lex }} B$ ) if either $A=B$, or $A \neq B$ and $n_{A}=n_{B}$ and for the least $i \in[\ell]$ such that $R_{i}(A) \neq R_{i}(B)$ the lexicographically first tuple $\bar{p}$ in the symmetric difference of $R_{i}(A)$ and $R_{i}(B)$ is contained in $R_{i}(B)$, or $n_{A}<n_{B}$.

\subsection{Definable Canonisation of Graphs of Bounded Rank Width}

Recall that our goal is to prove that FP+C captures PTIME on the class of graphs of rank width at most $k$. Towards this end we prove the following theorem.

Theorem 5.5. For every $k \geq 1$, the class of all graphs of rank width at most $k$ admits $\mathrm{FP}+\mathrm{C}-$ definable canonisation.

Observe that, combined with Lemma 5.4, this theorem implies Theorem 1.5.

The rest of this section is devoted to a proof of Theorem 5.5. Let us fix $k \geq 1$. Our strategy to define an ordered copy of a graph $G$ of rank width at most $k$ is similar to the proof strategy for showing that the Weisfeiler-Leman algorithm identifies such a graph. For ordered split pairs $(\bar{a}, \bar{b})$, flip functions $f$, and components $C$ of the flipped graph we recursively define an ordered 
copy of the induced subgraph $(G[C \cup \bar{a} \cup \bar{b}], \bar{a}, \bar{b})$. The first hurdle towards implementing this strategy is that we need to have explicit access to the flip function (this is different from the previous section where we only needed the existence of such a function in order to describe a strategy for Spoiler). However, we can not simply list all of the flip functions as there may be exponentially many. We remedy this by altering the definition of a flip so that, for every fixed $k$, there is only a polynomial number of flips.

Throughout this section let $k \geq 1$ be a fixed natural number. Let $G=(V, E)$ be a graph of rank width at most $k$ and let $n:=|V|$ denote the number of vertices of $G$. In this section an ordered split pair of order at most $k$ is simply a pair $(\bar{a}, \bar{b})$ where $\bar{a}, \bar{b} \in V \leq k$. For $v, w \in V$ we say that $v \approx_{(\bar{a}, \bar{b})} w$ if $N(v) \cap(\bar{a}, \bar{b})=N(w) \cap(\bar{a}, \bar{b})$. Clearly, $\approx_{(\bar{a}, \bar{b})}$ defines an equivalence relation on $V$. For tuples $\bar{a}, \bar{b} \in V \leq k$ we denote by $2^{\bar{a} \cup \bar{b}}$ the set of all subsets of $\bar{a} \cup \bar{b} \subseteq V$ where we interpret the tuples $\bar{a}$ and $\bar{b}$ as subsets of $V$. A flip extension of an ordered split pair $(\bar{a}, \bar{b})$ is a tuple

$$
\bar{s}=\left(\bar{a}, \bar{b}, f:\left(2^{\bar{a} \cup \bar{b}}\right)^{2} \rightarrow[n] \cup\{\perp\}\right)
$$

such that for all $M, N \in 2^{\bar{a} \cup \bar{b}}$ with $M \neq N$, either $f(M, N)=\perp$ or $f(N, M)=\perp$. For $v, w \in V$ we say that $v \approx_{\bar{s}} w$ if $v \approx_{(\bar{a}, \bar{b})} w$. We denote by $[v]_{\approx_{\bar{s}}}$ the equivalence class of $v$ with respect to $\approx_{\bar{s}}$. Moreover, we define the graph $G^{\bar{s}}=\left(V, E^{\bar{s}}, \bar{a}, \bar{b}\right)$ where

$$
\begin{aligned}
E^{\bar{s}}:= & \left\{v w \in E|f(N(v) \cap(\bar{a}, \bar{b}), N(w) \cap(\bar{a}, \bar{b}))=d \in[n] \wedge| N(v) \cap[w]_{\approx_{\bar{s}}} \mid<d\right\} \\
& \cup\left\{v w \notin E|f(N(v) \cap(\bar{a}, \bar{b}), N(w) \cap(\bar{a}, \bar{b}))=d \in[n] \wedge| N(v) \cap[w]_{\approx_{\bar{s}}} \mid \geq d\right\} .
\end{aligned}
$$

Finally we let $\operatorname{Comp}(G, \bar{s}) \subseteq 2^{V}$ be the set of vertex sets of the connected components of the graph $G^{\bar{s}}$ and for $v \in V$ we define $\operatorname{Comp}(G, \bar{s}, v)$ to be the unique $C \in \operatorname{Comp}(G, \bar{s})$ such that $v \in C$.

The following lemma is similar in nature to Lemma 3.6.

Lemma 5.6. Let $G$ be a graph and let $X \subseteq V(G)$. Furthermore, let $(\bar{a}, \bar{b})$ be an ordered split pair for $X$. Then there is a flip extension $\bar{s}=(\bar{a}, \bar{b}, f)$ such that $C \subseteq X$ or $C \subseteq \bar{X}$ for every $C \in \operatorname{Comp}(G, \bar{s})$.

The proof strategy for the lemma is similar to the proof of Lemma 3.6. Before giving the details, let us describe the main difference between the two proofs that also motivates our definition of a flip extension. Let $v, w \in V(G)$ and consider the sets $\widehat{P}:=[v]_{\widetilde{\sim}_{\bar{s}}}$ and $\widehat{Q}:=[w]_{\widetilde{\sim}_{\bar{s}}}$. As in the proof of Lemma 3.6, the bipartite graph between $\widehat{P} \cap X$ and $\widehat{Q} \cap \bar{X}$ is either empty or complete (using Lemma 3.3). In the latter case, we again wish to flip the edges between $\widehat{P} \cap X$ and $\widehat{Q} \cap \bar{X}$ in order to disconnect $X$ from $\bar{X}$. However, other than in the proof of Lemma 3.6, the bipartite graph between $\widehat{Q} \cap X$ and $\widehat{P} \cap \bar{X}$ may now be empty. To handle this particular case, the main idea is to use a degree-threshold $d$ for one of the two sides of the bipartite graph between $\widehat{P}$ and $\widehat{Q}$ to identify those vertices lying in $X$. In turn, this allows to identify the pairs $\left(v^{\prime}, w^{\prime}\right) \in \widehat{P} \times \widehat{Q}$ for which the edge relation needs to be flipped.

Proof. Let $M, N \subseteq \bar{a} \cup \bar{b}$, let $c(M):=\{v \in V(G) \mid N(v) \cap(\bar{a}, \bar{b})=M\}$ and similarly $c(N):=$ $\{v \in V(G) \mid N(v) \cap(\bar{a}, \bar{b})=N\}$. Let $P:=c(M) \cap X, \bar{P}:=c(M) \cap \bar{X}, Q:=c(N) \cap X$ and $\bar{Q}:=c(N) \cap \bar{X}$.

We need to define $f$ in such a way such that $(P \times \bar{Q}) \cap E\left(G^{\bar{s}}\right)=\emptyset$ and $(Q \times \bar{P}) \cap E\left(G^{\bar{s}}\right)=\emptyset$. If $(P \times \bar{Q}) \cap E(G)=\emptyset$ and $(Q \times \bar{P}) \cap E(G)=\emptyset$ then we can simply set $f(M, N)=n$ and $f(N, M)=\perp$. So assume one of the two sets is non-empty. Without loss of generality suppose $v^{\prime} \in Q$ and $w^{\prime} \in \bar{P}$ such that $v^{\prime} w^{\prime} \in E(G)$.

Claim 5.7. $Q \times \bar{P} \subseteq E(G)$.

Proof. Let $y \in Q$ and $z \in \bar{P}$. Then $v^{\prime} \approx_{X} y$ and $w^{\prime} \approx_{\bar{X}} z$ by Lemma 3.3. Hence,

$$
v^{\prime} w^{\prime} \in E(G) \Leftrightarrow v^{\prime} z \in E(G) \Leftrightarrow y z \in E(G) .
$$


If $(P \times \bar{Q}) \subseteq E(G)$ and $(Q \times \bar{P}) \subseteq E(G)$ then we can simply set $f(M, N)=1$ and $f(N, M)=\perp$. Hence, assume there are $v \in P$ and $w \in \bar{Q}$ such that $v w \notin E(G)$.

Claim 5.8. $(P \times \bar{Q}) \cap E(G)=\emptyset$.

Proof. Let $y \in P$ and $z \in \bar{Q}$. Then $v \approx_{X} y$ and $w \approx_{\bar{X}} z$ by Lemma 3.3. Hence,

$$
v w \in E(G) \Leftrightarrow v z \in E(G) \Leftrightarrow y z \in E(G) .
$$

Claim 5.9. The following inequalities hold:

(a) $\left|N\left(v^{\prime \prime}\right) \cap c(N)\right| \leq\left|N\left(w^{\prime \prime}\right) \cap c(N)\right|$ for all $v^{\prime \prime} \in P$ and $w^{\prime \prime} \in \bar{P}$.

(b) $\left|N\left(v^{\prime \prime \prime}\right) \cap c(M)\right| \geq\left|N\left(w^{\prime \prime \prime}\right) \cap c(M)\right|$ for all $v^{\prime \prime \prime} \in Q$ and $w^{\prime \prime \prime} \in \bar{Q}$.

Moreover, one of the two inequalities is strict.

Proof. We have $N\left(v^{\prime \prime}\right) \cap c(N) \subseteq Q \subseteq N\left(w^{\prime \prime}\right) \cap c(N)$ and $N\left(v^{\prime \prime \prime}\right) \cap c(M) \supseteq \bar{P} \supseteq N\left(w^{\prime \prime \prime}\right) \cap c(M)$ by Claim 5.7 and 5.8. This proves the inequalities. To argue that one of the two inequalities is strict suppose that $\left|N\left(v^{\prime \prime}\right) \cap c(N)\right|=\left|N\left(w^{\prime \prime}\right) \cap c(N)\right|$ for all $v^{\prime \prime} \in P$ and $w^{\prime \prime} \in \bar{P}$. Then $N\left(v^{\prime \prime}\right) \cap c(N)=Q$ for all $v^{\prime \prime} \in P$. But now $N\left(v^{\prime \prime \prime}\right) \cap c(M) \supsetneq \bar{P}$ for all $v^{\prime \prime \prime} \in Q$ since $v \in N\left(v^{\prime \prime \prime}\right)$. Thus, $\left|N\left(v^{\prime \prime \prime}\right) \cap c(M)\right|>\left|N\left(w^{\prime \prime \prime}\right) \cap c(M)\right|$ for all $v^{\prime \prime \prime} \in Q$ and $w^{\prime \prime \prime} \in \bar{Q}$.

Without loss of generality assume that the first inequality of the previous claim is strict. Let $d:=\min _{w^{\prime \prime} \in \bar{P}}\left|N\left(w^{\prime \prime}\right) \cap c(N)\right|$. We set $f(M, N):=d$ and $f(N, M):=\perp$. Then $\left|N\left(v^{\prime \prime}\right) \cap c(N)\right|<d$ for all $v^{\prime \prime} \in P$ and hence, $(P \times \bar{Q}) \cap E\left(G^{\bar{s}}\right)=\emptyset$ using Claim 5.8. Also $\left|N\left(w^{\prime \prime}\right) \cap c(N)\right| \geq d$ for all $w^{\prime \prime} \in \bar{P}$ and thus, $(Q \times \bar{P}) \cap E\left(G^{\bar{s}}\right)=\emptyset$ using Claim 5.7.

Lemma 5.10. Let $G$ be a graph, $X_{1} \subseteq X \subseteq V(G),(\bar{a}, \bar{b})$ an ordered split pair for $X$ and $\left(\bar{a}_{1}, \bar{b}_{1}\right)$ an ordered split pair for $X_{1}$ such that $X_{1} \cap \bar{a} \subseteq \bar{a}_{1}$. Let $v, w \in X_{1}$ such that $v \approx_{\left(\bar{a}_{1}, \bar{b}_{1}\right)} w$. Then $v \approx_{(\bar{a}, \bar{b})} w$.

Proof. Let $v, w \in X_{1}$ such that $v \approx_{\left(\bar{a}_{1}, \bar{b}_{1}\right)} w$. Then $v \approx_{X_{1}} w$ by Lemma 3.3. So $N(v) \cap \overline{X_{1}}=$ $N(w) \cap \overline{X_{1}}$ and $N(v) \cap\left(\bar{a}_{1}, \bar{b}_{1}\right)=N(w) \cap\left(\bar{a}_{1}, \bar{b}_{1}\right)$. Since $X_{1} \cap \bar{a} \subseteq \bar{a}_{1}$ and $X_{1} \cap \bar{b}=\emptyset$ this implies $v \approx_{(\bar{a}, \bar{b})} w$.

Lemma 5.11. Let $G$ be a graph, $X_{1}, X_{2} \subseteq V(G)$ such that $X_{1} \cap X_{2}=\emptyset,\left(\bar{a}_{1}, \bar{b}_{1}\right)$ an ordered split pair for $X_{1}$ and $\left(\bar{a}_{2}, \bar{b}_{2}\right)$ an ordered split pair for $X_{2}$ such that $X_{1} \cap \bar{b}_{2} \subseteq \bar{a}_{1}$. Let $v, w \in X_{1}$ such that $v \approx_{\left(\bar{a}_{1}, \bar{b}_{1}\right)} w$. Then $v \approx_{\left(\bar{a}_{2}, \bar{b}_{2}\right)} w$.

Proof. Let $v, w \in X_{1}$ such that $v \approx_{\left(\bar{a}_{1}, \bar{b}_{1}\right)} w$. Then $v \approx_{X_{1}} w$ by Lemma 3.3. So $N(v) \cap \overline{X_{1}}=$ $N(w) \cap \overline{X_{1}}$ and $N(v) \cap\left(\bar{a}_{1}, \bar{b}_{1}\right)=N(w) \cap\left(\bar{a}_{1}, \bar{b}_{1}\right)$. Since $X_{1} \cap \bar{a}_{2}=\emptyset$ and $X_{1} \cap \bar{b}_{2} \subseteq \bar{a}_{1}$ this implies $v \approx_{\left(\bar{a}_{2}, \bar{b}_{2}\right)} w$.

Definition 5.12. Let $G=(V, E)$ be a graph. Let $\bar{s}=(\bar{a}, \bar{b}, f)$ be a flip extension and let $C \subseteq V(G)$. An $\bar{s}$-anchored (ordered) copy of $C$ is a tuple $C_{\text {anc }}=\left(V_{\text {anc }}, E_{\text {anc }}, \bar{a}_{\text {anc }}, \bar{b}_{\text {anc }}, \eta\right)$ such that

(i) $V_{\text {anc }}$ is an initial segment of the nonnegative integers,

(ii) $\eta: V_{\text {anc }} \rightarrow\left(2^{\bar{a} \cup \bar{b}} \rightarrow \mathbb{N}\right)$, and

(iii) there is an isomorphism $\sigma:(G[C \cup \bar{a} \cup \bar{b}], \bar{a}, \bar{b}) \cong\left(V_{\text {anc }}, E_{\text {anc }}, \bar{a}_{\text {anc }}, \bar{b}_{\text {anc }}\right)$ such that $(\eta(i))(M)=$ $\left|N\left(\sigma^{-1}(i)\right) \cap\{w \in V(G) \mid N(w) \cap(\bar{a}, \bar{b})=M\}\right|$ for all $i \in V_{\text {anc }}$. 
In the following, this definition is typically applied to sets $C \in \operatorname{Comp}(G, \bar{s})$. More precisely, our aim is to define $\bar{s}$-anchored copies of $C$ for all $C \in \operatorname{Comp}(G, \bar{s})$ and all suitable flip extensions $\bar{s}$ in $\mathrm{FP}+\mathrm{C}$ in an inductive fashion. An important feature of an $\bar{s}$-anchored copy of $C$ is that, in addition to being an ordered copy of $C$, it records some "context information" on how the set $C$ is connected to the rest of the graph. This is the purpose of the function $\eta$. The context information will play a vital role in the proofs since it gives the relevant information to perform flips also in the $\bar{s}$-anchored copy of $C$.

Let us start by discussing how to represent the relevant objects in the logical framework. Since $k$ is a constant we can view a flip extension as a tuple $\bar{s} \in V(G)^{2 k} \times\{0, \ldots, n\}^{2^{2 k}}$ of fixed length. The first $2 k$ components represent the split pair $(\bar{a}, \bar{b})$ and the function $f: 2^{\bar{a} \cup \bar{b}} \rightarrow$ $[n] \cup\{\perp\}$ can be seen as a tuple in $\{0, \ldots, n\}^{2^{2 k}}$ where a 0 -entry is interpreted as $\perp$. Similar to the previous section, components (with respect to some flip extension) are represented by a single vertex from that component. To be more precise, a set $C \in \operatorname{Comp}(G, \bar{s})$ is represented by $\bar{s}$ and some $v \in C$. Observe that there is an FP+C-formula $\varphi(\bar{x}, y, z)$ such that $G=\varphi(\bar{s}, v, w)$ if and only if $w \in \operatorname{Comp}(G, \bar{s}, v)=C$ (see Example 5.1 for how to define reachability in $\mathrm{FP}+\mathrm{C}$ ).

To represent an $\bar{s}$-anchored copy $C_{\text {anc }}=\left(V_{\text {anc }}, E_{\text {anc }}, \bar{a}_{\text {anc }}, \bar{b}_{\text {anc }}, \eta\right)$ of a set $C \subseteq V(G)$, we represent the function $\eta$ by a relation $P_{\eta} \subseteq N(G)^{1+2^{2 k}}$ containing elements $\left(p, \bar{p}_{\eta}\right)$ for every $p \in V_{\text {anc }}$. The tuple $\bar{p}_{\eta} \in N(G)^{2^{2 k}}$ represents the function $\eta(p)$ and has an entry for each subset $M \subseteq \bar{a} \cup \bar{b}$ which specifies $(\eta(p))(M)$. Typically, we will denote the set $V_{\text {anc }} \subseteq N(G)$ by $P_{V}$, the relation $E_{\text {anc }} \subseteq N(G)^{2}$ by $P_{E}$, the tuple $\bar{a}_{\text {anc }}$ by $\bar{p}_{a}$, the tuple $\bar{b}_{\text {anc }}$ by $\bar{p}_{b}$, and the relation representing $\eta$ by $P_{\eta}$. Slightly abusing notation, we will write $C_{\text {anc }}=\left(P_{V}, P_{E}, \bar{p}_{a}, \bar{p}_{b}, \bar{P}_{\eta}\right)$. We can define such an anchored copy by FP+C-formulas $\psi_{V}(z), \psi_{E}\left(z_{1}, z_{2}\right), \psi_{\bar{a}}(\bar{z}), \psi_{\bar{b}}(\bar{z}), \psi_{\eta}\left(z, \bar{z}^{\prime}\right)$, where $z, z_{1}, z_{2}$ are number variables and $\bar{z}, \bar{z}^{\prime}$ are tuples of number variables of lengths $k, 2^{2 k}$, respectively.

We now start by constructing various $\mathrm{FP}+\mathrm{C}$ formulas. They will form the basic building blocks of the formulas defining $\bar{s}$-anchored copies of $C$ for sets $C \in \operatorname{Comp}(G, \bar{s})$.

Lemma 5.13. Let $G$ be a graph of rank width at most $k$. Also let $\bar{s}$ be a flip extension, $D \in$ $\operatorname{Comp}(G, \bar{s})$, and $D^{*}=\left(P_{V}, P_{E}, \bar{p}_{a}, \bar{p}_{b}, P_{\eta}\right)$ where $P_{V} \subseteq N(G), P_{E} \subseteq N(G)^{2}, \bar{p}_{a}, \bar{p}_{b} \in N(G)^{k}$ and $P_{\eta} \subseteq N(G)^{1+2^{2 k}}$.

There is an $\mathrm{FP}+\mathrm{C}$-sentence that, given access to the objects $\left(G, \bar{s}, D, D^{*}\right)$, decides if $D^{*}$ is an $\bar{s}$-anchored copy of $D$.

Proof. The proof of this lemma uses similar arguments as the proof of Lemma 5.3. Suppose $\bar{s}=(\bar{a}, \bar{b}, f)$ and let $R_{\eta}=\left\{\left(v, \bar{v}_{\eta}\right) \mid v \in V\right\}$ where $\bar{v}_{\eta} \in N(G)^{2^{2 k}}$ has an entry for each subset $M \subseteq \bar{a} \cup \bar{b}$ which specifies $|N(v) \cap\{w \in V(G) \mid N(w) \cap(\bar{a}, \bar{b})=M\}|$.

We need to check whether $\left(G[D], \bar{a}, \bar{b}, R_{\eta}\right) \cong D^{*}$. This can be achieved by implementing the Weisfeiler-Leman algorithm within fixed-point logic with counting. Here, the algorithm additionally needs to take the vertex-colouring into account that is given by $\left(\bar{a}, \bar{b}, R_{\eta}\right)$ and $\left(\bar{p}_{a}, \bar{p}_{b}, P_{\eta}\right)$, respectively

Given an $\bar{s}$-anchored copy $C_{\text {anc }}$ of a set $C \subseteq V(G)$, we regularly need to associate elements from $C_{\text {anc }}$ with their corresponding element in $C$ (via a bijection $\sigma$ according to Definition 5.12(iii)). Unfortunately, it is not possible to compute $\sigma$ directly within $\mathrm{FP}+\mathrm{C}$ as it may not be unique. The next lemma serves as tool that allows us to answer all necessary queries without directly accessing $\sigma$.

Lemma 5.14. Let $G$ be a graph, $X_{1} \subseteq X \subseteq V(G),(\bar{a}, \bar{b})$ an ordered split pair for $X$ and $\left(\bar{a}_{1}, \bar{b}_{1}\right)$ an ordered split pair for $X_{1}$. Let $\bar{s}=(\bar{a}, \bar{b}, f)$ and $\bar{s}_{1}=\left(\bar{a}_{1}, \bar{b}_{1}, f_{1}\right)$ be flip extensions. Moreover, suppose that $X_{1} \cap \bar{a} \subseteq \bar{a}_{1}$ and let $D \in \operatorname{Comp}\left(G, \bar{s}_{1}\right)$ such that $D \subseteq X_{1}$. Also let $D_{\text {anc }}$ be an $\bar{s}_{1}$-anchored copy of $D$ and let $\sigma$ be an isomorphism according to Definition 5.12(iii).

Given access to the objects $\left(G, \bar{s}_{1}, \bar{s}_{1}, D, D_{\mathrm{anc}}\right)$, the following queries can be defined using $\mathrm{FP}+\mathrm{C}$-formulas: 
(1) given $p \in V\left(D_{\text {anc }}\right)$, determine $N(v) \cap(\bar{a}, \bar{b})$ where $v=\sigma^{-1}(p)$,

(2) given $p \in V\left(D_{\mathrm{anc}}\right)$ and $v^{\prime} \in V(G)$, determine whether $v \approx_{\bar{s}} v^{\prime}$ where $v=\sigma^{-1}(p)$,

(3) given $p \in V\left(D_{\text {anc }}\right)$ and $w \in V(G)$, determine $\left|N(v) \cap[w]_{\approx_{\bar{s}}}\right|$ where $v=\sigma^{-1}(p)$, and

(4) given $p, q \in V\left(D_{\text {anc }}\right)$, determine whether $v w \in E\left(G^{\bar{s}}\right)$ where $v=\sigma^{-1}(p)$ and $w=\sigma^{-1}(q)$.

Just to be on the safe side, let us again explain the exact technical meaning of the assertions of the lemma, taking assertion (1) as an example. We need to construct an FP+C-formula $\varphi\left(\bar{x}, \bar{x}_{1}, y, Z_{V}, Z_{E}, \bar{z}_{a}, \bar{z}_{b}, Z_{\eta}, z, y^{\prime}\right)$, where $Z_{V}$ is a unary relation symbol of type 'number', $Z_{E}$ is a binary relation symbol of type 'number $\times$ number', $Z_{\eta}$ is a $\left(1+2^{2 k}\right)$-ary relation symbol of type ('number') ${ }^{\left(1+2^{2 k}\right)}, y, y^{\prime}$ are vertex variables, $z$ is a number variable, $\bar{z}_{a}, \bar{z}_{b}$ are $k$-tuples of number variables, and $\bar{x}, \bar{x}_{1}$ are tuples of individual variables of the type appropriate for representing flip extensions. The formula is supposed to have the following meaning. Suppose that $D_{\mathrm{anc}}=\left(P_{V}, P_{E}, \bar{p}_{a}, \bar{p}_{b}, P_{\eta}\right)$. Then for all $u \in D, p \in P_{V}$, and $w \in V(G)$,

$$
G \models \varphi\left(\bar{s}, \bar{s}_{1}, u, P_{V}, P_{E}, \bar{p}_{a}, \bar{p}_{b}, P_{\eta}, p, w\right)
$$

if and only if $w \in N(v) \cap(\bar{a}, \bar{b})$. We should think of the relations $P_{V}, P_{E}, P_{\eta}$, which determine the core of the structure $D_{\text {anc }}$, as being defined earlier in some inductive process. Note that we do not specify $D$ explicitly in the definition, but only implicitly by giving the flip extension $\bar{s}_{1}$ and the vertex $u$.

Proof of Lemma 5.14. Let $p \in V\left(D_{\text {anc }}\right)$ and let $v=\sigma^{-1}(p)$. Given $p$, one can clearly find some $v^{\prime \prime} \in D$ such that $v \approx_{\bar{s}_{1}} v^{\prime \prime}$. By Lemma 5.10 we conclude that $v \approx_{\bar{s}} v^{\prime \prime}$. Using $v^{\prime \prime}$ instead of $v$ one can already solve Tasks (1) and (2).

So consider Task (3). First note that we can easily define the set $[w]_{\approx_{\bar{s}}}$ given $w$, and $\bar{a}, \bar{b}$, which are both contained in $\bar{s}$. Now let

$$
Y_{1}=\bigcup_{u \in D: u \approx_{\bar{s}_{1}} v^{\prime \prime}} N(u) \triangle N\left(v^{\prime \prime}\right)
$$

(here $P \triangle Q$ denotes the symmetric difference between the two sets $P$ and $Q$ ). Then $Y_{1} \subseteq X_{1}$ and $N(v) \cap \overline{Y_{1}}=N\left(v^{\prime \prime}\right) \cap \overline{Y_{1}}$ by Lemma 3.3. Now we compute

$$
\begin{aligned}
& \left|N(v) \cap[w]_{\approx_{\bar{s}}}\right|=\left|N(v) \cap[w]_{\approx_{\bar{s}}} \cap Y_{1}\right|+\left|N(v) \cap[w]_{\approx_{\bar{s}}} \cap \overline{Y_{1}}\right| \\
& =\left|N(v) \cap[w]_{\approx_{\bar{s}}} \cap Y_{1}\right|+\left|N\left(v^{\prime \prime}\right) \cap[w]_{\approx_{\bar{s}}} \cap \overline{Y_{1}}\right| .
\end{aligned}
$$

The second term can be computed easily, so we only have to determine $\left|N(v) \cap[w]_{\approx_{\bar{s}}} \cap Y_{1}\right|$. For $M \subseteq\left(\bar{a}_{1}, \bar{b}_{1}\right)$ we define $c(M):=\left\{u \in V(G) \mid N(u) \cap\left(\bar{a}_{1}, \bar{b}_{1}\right)=M\right\}$. We have that

$$
\left|N(v) \cap[w]_{\approx_{\bar{s}}} \cap Y_{1}\right|=\sum_{M \subseteq\left(\bar{a}_{1}, \bar{b}_{1}\right)}\left|N(v) \cap[w]_{\approx_{\bar{s}}} \cap Y_{1} \cap c(M)\right| .
$$

Using the fact that $Y_{1} \subseteq X_{1}$ and Lemma 5.10 we get that

$$
[w]_{\approx_{\bar{s}}} \cap c(M) \cap Y_{1} \neq \emptyset \Rightarrow[w]_{\approx_{\bar{s}}} \cap c(M) \cap Y_{1}=c(M) \cap Y_{1} .
$$

Hence,

$$
\left|N(v) \cap[w]_{\approx_{\bar{s}}} \cap Y_{1}\right|=\sum_{\substack{M \subseteq\left(\bar{a}_{1}, \bar{b}_{1}\right): \\[w]_{\tau_{\bar{s}}} \cap c(M) \cap Y_{1} \neq \emptyset}}\left|N(v) \cap Y_{1} \cap c(M)\right| .
$$

But $\left|N(v) \cap Y_{1} \cap c(M)\right|=|N(v) \cap c(M)|-\left|N(v) \cap c(M) \cap \overline{Y_{1}}\right|=|N(v) \cap c(M)|-\left|N\left(v^{\prime \prime}\right) \cap c(M) \cap \overline{Y_{1}}\right|$. Recalling $|N(v) \cap c(M)|=(\eta(p))(M)$, the last term is easy to compute.

Finally note that Task (4) can be solved using the first three results in order to determine $N(v) \cap(\bar{a}, \bar{b}), N(w) \cap(\bar{a}, \bar{b})$ and $\left|N(v) \cap[w]_{\approx_{\bar{s}}}\right|$. 
Remark 5.15. In later proofs we wish to apply the lemma to sets $D \in \operatorname{Comp}\left(G, \bar{s}_{1}\right)$ without exactly knowing whether $D \subseteq X_{1}$. However, we do not need to know whether $D \subseteq X_{1}$ in order to determine whether the lemma is applicable. One of the crucial steps in the proof of the previous lemma is to obtain Equation (1). Indeed, besides being able to apply Lemma 5.10, this is the only place where we need that $D \subseteq X_{1}$. Since we can easily check within FP+C whether Equation (1) holds, we can also find an FP+C-formula that checks whether the lemma can be applied given $\left(\bar{s}, \bar{s}_{1}, u\right)$ where $u \in D$ is arbitrary.

We also need the following variant of the previous lemma.

Lemma 5.16. Let $G$ be a graph, $X_{1}, X_{2} \subseteq V(G)$ such that $X_{1} \cap X_{2}=\emptyset,\left(\bar{a}_{1}, \bar{b}_{1}\right)$ an ordered split pair for $X_{1}$ and $\left(\bar{a}_{2}, \bar{b}_{2}\right)$ an ordered split pair for $X_{2}$. Let $\bar{s}_{1}=\left(\bar{a}_{1}, \bar{b}_{1}, f_{1}\right)$ and $\bar{s}_{2}=\left(\bar{a}_{2}, \bar{b}_{2}, f_{2}\right)$ be flip extensions. Moreover, suppose that $X_{1} \cap \bar{b}_{2} \subseteq \bar{a}_{1}$ and let $D \in \operatorname{Comp}\left(G, \bar{s}_{1}\right)$ such that $D \subseteq X_{1}$. Also let $D_{\text {anc }}$ be an $\bar{s}_{1}$-anchored copy of $D$ and let $\sigma$ denote any isomorphism according to Definition 5.12(iii).

Given access to the objects $\left(G, \bar{s}_{1}, \bar{s}_{2}, D, D_{\text {anc }}\right)$, the following queries can be defined using $\mathrm{FP}+\mathrm{C}$-formulas:

(1) given $p \in V\left(D_{\text {anc }}\right)$, determine $N(v) \cap\left(\bar{a}_{2}, \bar{b}_{2}\right)$ where $v=\sigma^{-1}(p)$,

(2) given $p \in V\left(D_{\mathrm{anc}}\right)$ and $v^{\prime} \in V(G)$, determine whether $v \approx_{\bar{s}_{2}} v^{\prime}$ where $v=\sigma^{-1}(p)$,

(3) given $p \in V\left(D_{\text {anc }}\right)$ and $w \in V(G)$, determine $\left|N(v) \cap[w]_{\approx_{\bar{s}_{2}}}\right|$ where $v=\sigma^{-1}(p)$, and

(4) given $p, q \in V\left(D_{\text {anc }}\right)$, determine whether $v w \in E\left(G^{\bar{s}_{2}}\right)$ where $v=\sigma^{-1}(p)$ and $w=\sigma^{-1}(q)$.

Proof. Analogous to the proof of Lemma 5.14 using Lemma 5.11 instead of Lemma 5.10.

Recall the definition of nice triples of ordered split pairs (see Definition 4.1).

Lemma 5.17. Let $G$ be a graph and $X, X_{1}, X_{2} \subseteq V(G)$ such that $X=X_{1} \uplus X_{2}$. Let $(\bar{a}, \bar{b})$ be an ordered split pair for $X$ and let $\left(\bar{a}_{i}, \bar{b}_{i}\right)$ be ordered split pairs for $X_{i}, i \in\{1,2\}$, that are nice with respect to $(\bar{a}, \bar{b})$. Moreover, let $\bar{s}=(\bar{a}, \bar{b}, f)$ and $\bar{s}_{i}=\left(\bar{a}_{i}, \bar{b}_{i}, f_{i}\right), i \in\{1,2\}$, be flip extensions. Let $C \in \operatorname{Comp}(G, \bar{s})$ such that $C \subseteq X$, and let $\mathcal{D}_{i} \subseteq \operatorname{Comp}\left(G, \bar{s}_{i}\right)$ such that

$$
X_{i} \subseteq \bigcup_{D \in \mathcal{D}_{i}} D
$$

For every $D \in \mathcal{D}_{i}$ let $D_{\text {anc }}$ be an $\bar{s}_{i}$-anchored copy of $D$ and let $\mathcal{D}_{i}^{\text {anc }}$ be the set of all of those copies for $i \in\{1,2\}$.

Then in $\mathrm{FP}+\mathrm{C}$, given access to $\left(G, \bar{s}, \bar{s}_{1}, \bar{s}_{2}, C, \mathcal{D}_{1}, \mathcal{D}_{1}^{\text {anc }}, \mathcal{D}_{2}, \mathcal{D}_{2}^{\text {anc }}\right)$, we can define an $\bar{s}$-anchored copy $C_{\text {anc }}$ of $C$.

Let us again discuss the precise meaning of this statement. Specifically, we need to elaborate on how to represent the families of sets $\mathcal{D}_{i}$ and the families of $\bar{s}_{i}$-anchored copies $\mathcal{D}_{i}^{\text {anc }}$. We index the two families by elements $u \in V(G)$. For every $u \in V(G)$ we let $D_{i, u} \in \operatorname{Comp}\left(G, \bar{s}_{i}\right)$ be the unique component with $u \in D_{i, u}$. If $D_{i, u} \in \mathcal{D}_{i}$, we denote its anchored copy in $\mathcal{D}_{i}^{\text {anc }}$ by $D_{i, u}^{\text {anc }}$. Note, however, that we do not necessarily have $D_{i, u} \in \mathcal{D}_{i}$ for all $u$. The only requirement is that $X_{i}$ is a subset of the union of all $D_{i, u}$ in $\mathcal{D}$. We represent the family $\mathcal{D}_{i}^{\text {anc }}$ by a binary relation $P_{i, V} \subseteq V(G) \times N(G)$, a ternary relation $P_{i, E} \subseteq V(G) \times N(G)^{2},(k+1)$-ary relations $P_{i, \bar{a}}, P_{i, \bar{b}} \subseteq V(G) \times N(G)^{k}$, and a $\left(2^{2 k}+2\right)$-ary relation $P_{i, \eta}$ such that if $u \in V(G)$ with $D_{i, u} \in \mathcal{D}$ then $D_{i, u}^{\text {anc }}=(V, E, \bar{a}, \bar{b}, \eta)$ where

- $V=\left\{p \in N(G) \mid(u, p) \in P_{i, V}\right\}$;

- $E=\left\{(p, q) \in N(G)^{2} \mid(u, p, q) \in P_{i, E}\right\}$; 
- $\bar{a} \in N(G)^{k}$ is the unique tuple with $(u, \bar{a}) \in P_{i, \bar{a}}$;

- $\bar{b} \in N(G)^{k}$ is the unique tuple with $(u, \bar{b}) \in P_{i, \bar{b}}$;

- $\eta$ is represented by $P_{\eta} \subseteq N(G)^{1+2^{2 k}}$ where $P_{\eta}=\left\{\bar{p} \in N(G)^{1+2^{2 k}} \mid(u, \bar{p}) \in P_{i, \eta}\right\}$.

To define $C_{\text {anc }}$, we need to construct FP+C-formulas $\varphi_{V}, \varphi_{E}, \varphi_{\bar{a}}, \varphi_{\bar{b}}, \varphi_{\eta}$. They all have free variables $\bar{x}, \bar{x}_{1}, \bar{x}_{2}$ for the flip extensions $\bar{s}, \bar{s}_{1}, \bar{s}_{2}$, a free vertex variable $y$ for an element of the component $C$, and for $i=1,2$ free relation variables $Z_{i, V}, Z_{i, E}, Z_{i, a}, Z_{i, b}, Z_{i, \eta}$ for the family $\mathcal{D}_{i}^{\text {anc }}$. The family $\mathcal{D}_{i}$ is only specified implicitly: $\mathcal{D}_{i}$ consists of all components $D \in \operatorname{Comp}\left(G, \bar{s}_{i}\right)$ such that a $u \in D$ appears as an index of a structure in $\mathcal{D}_{i}^{\text {anc }}$, that is, as the first component of a tuple in the relations $Z_{i, V}, Z_{i, E}, Z_{i, a}, Z_{i, b}, Z_{i, \eta}$. In addition, the formula $\varphi_{V}$ has a free number variable $z$ for the elements of $V\left(C_{\text {anc }}\right)$. The formula $\varphi_{E}$ has two free number variables $z_{1}, z_{2}$ for the elements of $E\left(C_{\text {anc }}\right)$. The formula $\varphi_{\bar{a}}, \varphi_{\bar{b}}$ have a $k$-tuple of free number variables $\bar{z}$ for $\bar{a}_{\text {anc }}, \bar{b}_{\text {anc }}$, respectively. And finally, the formula $\varphi_{\eta}$ has a $\left(1+2^{2 k}\right)$-tuple of free number variables $\bar{z}$ to specify the function $\eta$ of $C_{\text {anc }}$.

Proof of Lemma 5.17. First, we can assume without loss of generality that we can apply Lemma 5.14 and 5.16 to every $D \in \mathcal{D}_{i}$ (by eliminating all sets that do not satisfy the requirements, see Remark 5.15).

Let $i \in\{1,2\}$. Let $D \in \mathcal{D}_{i}$ and let $D_{\text {anc }} \in \mathcal{D}_{i}^{\text {anc }}$ be the $\bar{s}_{i}$-anchored copy of $D$. We define $D_{\text {anc }}^{\bar{s}}=\left(V\left(D_{\text {anc }}\right), E^{\bar{s}}\right)$ where $E^{\bar{s}}=\left\{i j \mid \sigma_{D}^{-1}(i) \sigma_{D}^{-1}(j) \in E\left(G^{\bar{s}}\right)\right\}$ and $\sigma_{D}$ is an isomorphism to the $\bar{s}_{i}$-anchored copy according to Definition 5.12(iii). Note that we can define $D_{\text {anc }}^{\bar{s}}$ in FP+C by Lemma 5.14(4). Now let $D^{\prime} \subseteq D$ be a connected component of $\left(G^{\bar{s}}\right)[D]$. Then $D^{\prime} \subseteq C$ or $D^{\prime} \cap C=\emptyset$. Also $\sigma_{D}\left(D^{\prime}\right)$ is a connected component of $D_{\text {anc }}^{\bar{s}}$. Since we can match the connected components of $\left(G^{\bar{s}}\right)[D]$ to those of $D_{\text {anc }}^{\bar{s}}$ by implementing the Weisfeiler-Leman algorithm in fixed-point logic with counting (see Lemma 5.3), we can define an $\bar{s}_{i}$-anchored copy $D_{\text {anc }}^{C}$ of $D \cap C$. Let $C_{i}=C \cap \bigcup_{D \in \mathcal{D}_{i}} D$. By lexicographically ordering the $\bar{s}_{i}$-anchored copies of the sets $D \cap C, D \in \mathcal{D}_{i}$, we can also define an $\bar{s}_{i}$-anchored copy $C_{i}^{\text {anc }}$ of $C_{i}$. Note that we can figure out whether there is an edge between two vertices of different components by looking at the $\eta$ functions. Indeed, in order to know whether there is an edge between $p \in D$ and $q \in D^{\prime}$ we need to be able to compute, for $v=\sigma_{D}^{-1}(p)$ and $w=\sigma_{D^{\prime}}^{-1}(q)$, the sets $N(v) \cap\left(\bar{a}_{i}, \bar{b}_{i}\right), N(w) \cap\left(\bar{a}_{i}, \bar{b}_{i}\right)$ and the value $\left|N(v) \cap[w]_{\widetilde{s}_{i}}\right|$. Then the flip extension $\bar{s}_{i}$ tells us whether there is an edge or not. The neighbourhoods to $\left(\bar{a}_{i}, \bar{b}_{i}\right)$ are directly given in the anchored copy and the number of neighbours in some given equivalence class is stored in the $\eta$-function.

Essentially repeating this process applying Lemma 5.16 instead of Lemma 5.14 we can also compute an $\bar{s}_{2}$-anchored copy $C_{2 \backslash 1}^{\text {anc }}$ of the set $C_{2} \backslash C_{1}$.

Next, we can turn the $\bar{s}_{1}$-anchored copy $C_{1}^{\text {anc }}$ of $C_{1}$ into an $\bar{s}$-anchored copy $C_{1}^{\text {anc, } \bar{s}}$ of $C_{1}$ using Lemma 5.14, Item (1) and (3). Similarly, we can turn the $\bar{s}_{2}$-anchored copy $C_{2 \backslash 1}^{\text {anc }}$ of $C_{2} \backslash C_{1}$ into an $\bar{s}$-anchored copy $C_{2 \backslash 1}^{\text {anc, } \bar{s}}$ of $C_{1} \backslash C_{2}$.

Finally, in order to obtain $C_{\text {anc }}$, we take the disjoint union of $C_{1}^{\text {anc, }, \bar{s}}$ and $C_{2 \backslash 1}^{\text {anc, }, \bar{s}}$ (where $C_{1}^{\text {anc, }, \bar{s}}$ comes first, i.e. the corresponding vertices get smaller numbers assigned than the vertices from $\left.C_{2 \backslash 1}^{\text {anc, }, \bar{s}}\right)$. It only remains to recover the edges between the two sides. But this can be done using Lemma 5.16, Item (1) and (3) reconstructing the information whether such a given edge is flipped by $\bar{s}_{1}$.

With this, we are ready to prove our theorem.

Proof of Theorem 5.5. Let $G$ be a graph of rank width at most $k$. We will inductively construct $\bar{s}$-anchored copies $C_{\text {anc }}$ for flip extensions $\bar{s}$ and components $C \in \operatorname{Comp}(G, \bar{s})$, using Lemma 5.17 in the inductive step. The base step for components $C$ consisting of a single element will be easy. 
To describe the proof, we fix $G$, but of course the $\mathrm{FP}+\mathrm{C}$-formulas we shall construct will not depend on the specific graph $G$ and will work for every graph of rank width at most $k$.

Note first that the set of all flip extensions of $G$, viewed as tuples in $V(G)^{2 k} \times N(G)^{2^{2 k}}$, is definable in FP+C: we only need to make sure that the part of the tuple in $N(G)^{2^{2 k}}$ representing the flip function adheres to the simple conditions in the definition of a flip extension. Let $F(G) \subseteq V(G)^{2 k} \times N(G)^{2^{2 k}}$ be the set of all flip extensions.

In our main induction, to be implemented by an $\mathrm{FP}+\mathrm{C}$-formula, we will define an increasing collection of anchored copies of components $C \in \operatorname{Comp}(G, \bar{s})$ for flip extensions $\bar{s}$. We shall simultaneously define five relations for every $\ell \geq 1$. Recall that for a flip extension $\bar{s}$ and $u \in V(G)$ we denote by $\operatorname{Comp}(G, \bar{s}, u)$ the unique $C \in \operatorname{Comp}(G, \bar{s})$ such that $u \in C$.

- $R^{(\ell)} \subseteq F(G) \times V(G)$ will consist of those tuples $(\bar{s}, u)$ such that we have already defined an anchored copy $C_{\text {anc }}$ of the component $\operatorname{Comp}(G, \bar{s}, u)$;

- $R_{V}^{(\ell)} \subseteq F(G) \times V(G) \times N(G)$ will consist of all tuples $(\bar{s}, u, p)$ such that $(\bar{s}, u) \in R^{(\ell)}$ and $p \in V\left(C_{\text {anc }}\right)$ for the anchored copy $C_{\text {anc }}$ of the component $\operatorname{Comp}(G, \bar{s}, u)$;

- $R_{E}^{(\ell)} \subseteq F(G) \times V(G) \times N(G)^{2}$ will consist of all tuples $\left(\bar{s}, u, p_{1}, p_{2}\right)$ such that $(\bar{s}, u) \in R^{(\ell)}$ and $\left(p_{1}, p_{2}\right) \in E\left(C_{\text {anc }}\right)$ for the anchored copy $C_{\text {anc }}$ of the component $\operatorname{Comp}(G, \bar{s}, u)$;

- $R_{\bar{a}}^{(\ell)} \subseteq F(G) \times V(G) \times N(G)^{k}$ will consist of all tuples $(\bar{s}, u, \bar{p})$ such that $(\bar{s}, u) \in R^{(\ell)}$ and $\bar{p}=\bar{a}_{\text {anc }}$ for the anchored copy $C_{\text {anc }}$ of the component $\operatorname{Comp}(G, \bar{s}, u)$;

- $R_{\bar{b}}^{(\ell)} \subseteq F(G) \times V(G) \times N(G)^{k}$ will consist of all tuples $(\bar{s}, u, \bar{p})$ such that $(\bar{s}, u) \in R^{(\ell)}$ and $\bar{p}=\bar{b}_{\text {anc }}$ for the anchored copy $C_{\text {anc }}$ of the component $\operatorname{Comp}(G, \bar{s}, u)$;

- $R_{\eta}^{(\ell)} \subseteq F(G) \times V(G) \times N(G)^{1+2^{2 k}}$ will consist of all tuples $(\bar{s}, u, \bar{p})$ such that $(\bar{s}, u) \in R^{(\ell)}$ and $\bar{p} \in R_{\eta}$ where $R_{\eta}$ represents the function $\eta$ of the anchored copy $C_{\text {anc }}$ of the component $\operatorname{Comp}(G, \bar{s}, u)$.

In the $\mathrm{FP}+\mathrm{C}$-formula that we construct, the relations will be represented by relation variables $X, X_{V}, X_{E}, X_{\bar{a}}, X_{\bar{b}}, X_{\eta}$ of appropriate types. $R^{(\ell)}$ will be the value of $X$ after the $\ell$ th iteration of the main fixed-point iteration (and similarly for the other variables).

In the base step of our induction, we define $R^{(1)}$ to consist of all tuples $(\bar{s}, u) \in F(G) \times V(G)$ such that $\{u\} \in \operatorname{Comp}(G, \bar{s})$. Then defining the relations $R_{V}^{(1)}, \ldots, R_{\eta}^{(1)}$, that is, the anchored copy of $\{u\}$, is easy, because the anchored copy only has a constant number of elements, namely vertices corresponding to $u$ and to the vertices from the split pair $(\bar{a}, \bar{b})$ of the flip extension $\bar{s}$.

So let us turn to the inductive step. We have already defined relations $R^{(\ell)}, R_{V}^{(\ell)}, \ldots, R_{\eta}^{(\ell)}$. We look at a flip extension $\bar{s}$ and a vertex $u \in V(G)$ such that $(\bar{s}, u) \notin R^{(\ell)}$, that is, we have not yet defined an anchored copy of the component $C \in \operatorname{Comp}(G, \bar{s})$ that contains $u$. For all $\bar{s}_{1}, \bar{s}_{2} \in F(G)$, we do the following. We let $\mathcal{D}_{i}$ be the set of all $D \in \operatorname{Comp}\left(G, \bar{s}_{i}\right)$ such that $\left(\bar{s}_{i}, v\right) \in R^{(\ell)}$ for some $v \in D$. By induction, this means that actually $\left(\bar{s}_{i}, v\right) \in R^{(\ell)}$ for all $v \in D$ and that we have already computed an anchored copy $D_{\text {anc }}$ of $D$, which is represented by the $\left(\bar{s}_{i}, v\right)$-entries of the relations in $R_{V}^{(\ell)}, \ldots, R_{\eta}^{(\ell)}$. We let $\mathcal{D}_{i}^{\text {anc }}$ be the set of all these anchored copies $D_{\text {anc }}$. Now we apply the FP+C-formulas of Lemma 5.17 to $\left(G, \bar{s}, \bar{s}_{1}, \bar{s}_{2}, C, \mathcal{D}_{1}, \mathcal{D}_{1}^{\text {anc }}, \mathcal{D}_{2}, \mathcal{D}_{2}^{\text {anc }}\right)$. We obtain a structure $C_{\bar{s}_{1}, \bar{s}_{2}}=\left(P_{V}, P_{E}, \bar{p}_{a}, \bar{p}_{b}, P_{\eta}\right)$. Note that $C_{\bar{s}_{1}, \bar{s}_{2}}$ is not necessarily an $\bar{s}$ anchored copy of $C$, because we do not know whether there are sets $X, X_{1}, X_{2}$ such that $\bar{s}, \bar{s}_{1}, \bar{s}_{2}$ satisfy the assumptions of Lemma 5.17. However, using Lemma 5.13, we can check if $C_{\bar{s}_{1}, \bar{s}_{2}}$ is an anchored copy, regardless of whether the assumptions of Lemma 5.17 are satisfied. If $C_{\bar{s}_{1}, \bar{s}_{2}}$ is an $\bar{s}$-anchored copy of $C$, we call $\left(\bar{s}_{1}, \bar{s}_{2}\right) \operatorname{good}$ for $\bar{s}$.

If there are $\bar{s}_{1}, \bar{s}_{2} \in F(G)$ that are good for $\bar{s}$, we add $(\bar{s}, u)$ to $R^{(\ell+1)}$. We let $C_{\text {anc }}=$ $\left(P_{V}, P_{E}, \bar{p}_{a}, \bar{p}_{b}, P_{\eta}\right)$ be the lexicographically smallest of all structures $C_{\bar{s}_{1}, \bar{s}_{2}}$, and we add 
- all tuples $(\bar{s}, u, p)$ for $p \in P_{V}$ to $R_{V}^{(\ell+1)}$;

- all tuples $\left(\bar{s}, u, p_{1}, p_{2}\right)$ for $\left(p_{1}, p_{2}\right) \in P_{E}$ to $R_{E}^{(\ell+1)}$;

- the tuple $\left(\bar{s}, u, \bar{p}_{a}\right)$ to $R_{\bar{a}}^{(\ell+1)}$;

- the tuple $\left(\bar{s}, u, \bar{p}_{b}\right)$ to $R_{\bar{b}}^{(\ell+1)}$;

- the tuple $(\bar{s}, u, \bar{p})$ for $\bar{p} \in P_{\eta}$ to $R_{\eta}^{(\ell+1)}$.

This completes the description of the inductive construction.

It is not yet clear what the inductive process actually achieves, because it is not clear that in the inductive step we find any good tuples. To prove that the inductive process will eventually produce an ordered copy of $G$, we take a branch decomposition $(T, \gamma)$ of $G$ of width $k$. We prove that for every node $t \in V(T)$, every flip extension $\bar{s}$ for the set $X=\gamma(t)$, and every component $C \in \operatorname{Comp}(G, \bar{s})$ such that $C \subseteq X$, there is an $\ell \geq 1$ such that $(\bar{s}, u) \in R^{(\ell)}$ for all $u \in C$. Indeed, we can choose $\ell$ to be 1 plus the depth of $t$ in the tree, that is, the maximum length of a (directed) path from $t$ to a leaf.

The proof is by induction on $T$. The base step is trivial, because for leaves $t$, we have $|\gamma(t)|=1$. For the inductive step $\ell \rightarrow \ell+1$, let $t$ be a node of depth $\ell+1$ with children $t_{1}, t_{2}$, and let $X=\gamma(t)$ and $X_{i}=\gamma\left(t_{i}\right)$. Then $X_{1} \cup X_{2}=X$ and $X_{1} \cap X_{2}=\emptyset$. Let $(\bar{a}, \bar{b})$ be an ordered split pair for $X$ and $\bar{s}$ a flip extension of $(\bar{a}, \bar{b})$. Then, by Lemma 4.2, there exist ordered split pairs $\left(\bar{a}_{i}, \bar{b}_{i}\right)$ for $X_{i}$ that are nice with respect to $(\bar{a}, \bar{b})$. Moreover, by Lemma 5.6 we can choose a flip extension $\bar{s}_{i}$ of $\left(\bar{a}_{i}, \bar{b}_{i}\right)$ such that for every component $D \in \operatorname{Comp}\left(G, \bar{s}_{i}\right)$, either $D \subseteq X_{i}$ or $D \cap X_{i}=\emptyset$. Let $\mathcal{D}_{i}$ be the set of all components $D \in \operatorname{Comp}\left(G, \bar{s}_{i}\right)$ such that $\left(\bar{s}_{i}, v\right) \in R_{i}^{(\ell)}$ for all $v \in D$. By the induction hypothesis, for every component $D \in \operatorname{Comp}\left(G, \bar{s}_{i}\right)$ with $D \subseteq X_{i}$ we have $D \in \mathcal{D}_{i}$. This implies, by Lemma 5.17 , that the pair $\left(\bar{s}_{1}, \bar{s}_{2}\right)$ is good for $\bar{s}$. But then $(\bar{s}, u) \in R^{(\ell+1)}$ for every $u \in C$.

There is a small problem at the root $r$ of $T$ because for $X=\gamma(r)=V(G)$ there is no split tuple. However, we can apply the same construction as in the inductive step with $\bar{s}$ being the empty tuple. The problem is only a syntactic one: inductions formalised in FP+C can only define relations of a fixed type, so we cannot directly replace the $2 k+2^{2 k}$ tuple $\bar{s}$ by the empty tuple. To resolve this, we carry out the last step of the inductive process separately adapting the types accordingly. In the end, we obtain the desired ordered copy of $G$.

\section{Conclusions}

In this paper we considered the isomorphism and canonisation problem for graphs of bounded rank width. The first main result is that the Weisfeiler-Leman dimension of graphs of rank width at most $k$ is at most $3 k+4$, that is, the $(3 k+4)$-dimensional Weisfeiler-Leman algorithm identifies all graphs of rank width at most $k$. This implies that isomorphism testing and canonisation for graphs of rank width at most $k$ can be done in time $n^{O(k)}$.

The second main result is that fixed-point logic with counting captures polynomial time on the class of graphs of rank width at most $k$.

We remark that it is not difficult to obtain an $\Omega(k)$ lower bound on the Weisfeiler-Leman dimension of graphs of rank width $k$. Actually, combining a recent lower bound on the WeisfeilerLeman dimension for graphs of bounded tree width [32] and Theorem 2.2, the Weisfeiler-Leman dimension of graphs of rank width at most $k$ is at least $\left\lfloor\frac{k}{2}\right\rfloor-2$. Thus our upper bound is asymptotically tight up to a factor of six. Naturally, it would be nice to close or further narrow the gap between the upper and lower bound. 
A more important question is whether isomorphism testing is also fixed-parameter tractable when parameterized by rank width. We remark that fpt algorithms for isomorphism testing parameterized by tree width are known [36, 23].

An interesting open question on the logical side is whether rank decompositions can be defined in monadic second order logic. A partial result for graphs of bounded linear clique width has been obtained in [4]. We believe the techniques developed in this paper might also prove helpful for resolving the general question.

\section{References}

[1] Matthew Anderson, Anuj Dawar, and Bjarki Holm. Solving linear programs without breaking abstractions. J. ACM, 62(6):48:1-48:26, 2015. doi:10.1145/2822890.

[2] László Babai. Graph isomorphism in quasipolynomial time [extended abstract]. In Daniel Wichs and Yishay Mansour, editors, Proceedings of the 48th Annual ACM SIGACT Symposium on Theory of Computing, STOC 2016, Cambridge, MA, USA, June 18-21, 2016, pages 684-697. ACM, 2016. doi:10.1145/2897518.2897542.

[3] Hans L. Bodlaender. Polynomial algorithms for graph isomorphism and chromatic index on partial k-trees. J. Algorithms, 11(4):631-643, 1990. doi :10.1016/0196-6774(90)90013-5.

[4] Mikolaj Bojanczyk, Martin Grohe, and Michal Pilipczuk. Definable decompositions for graphs of bounded linear cliquewidth. Log. Methods Comput. Sci., 17(1), 2021. doi: 10.23638/LMCS-17(1:5) 2021.

[5] Jin-yi Cai, Martin Fürer, and Neil Immerman. An optimal lower bound on the number of variables for graph identification. Comb., 12(4):389-410, 1992. doi:10.1007/BF01305232.

[6] Ashok K. Chandra and David Harel. Structure and complexity of relational queries. J. Comput. Syst. Sci., 25(1):99-128, 1982. doi:10.1016/0022-0000(82)90012-5.

[7] Bruno Courcelle. The monadic second-order logic of graphs VIII: orientations. Ann. Pure Appl. Logic, 72(2):103-143, 1995. doi:10.1016/0168-0072(95)94698-V.

[8] Bruno Courcelle and Joost Engelfriet. A logical characterization of the sets of hypergraphs defined by hyperedge replacement grammars. Math. Syst. Theory, 28(6):515-552, 1995.

[9] Bruno Courcelle, Joost Engelfriet, and Grzegorz Rozenberg. Handle-rewriting hypergraph grammars. J. Comput. Syst. Sci., 46(2):218-270, 1993. doi:10.1016/0022-0000(93) 90004-G.

[10] Bruno Courcelle, Johann A. Makowsky, and Udi Rotics. Linear time solvable optimization problems on graphs of bounded clique-width. Theory Comput. Syst., 33(2):125-150, 2000. doi: $10.1007 / \mathrm{s} 002249910009$.

[11] Bruno Courcelle and Stephan Olariu. Upper bounds to the clique width of graphs. Discrete Applied Mathematics, 101(1-3):77-114, 2000. doi:10.1016/S0166-218X (99)00184-5.

[12] Heinz-Dieter Ebbinghaus and Jörg Flum. Finite model theory. Perspectives in Mathematical Logic. Springer-Verlag, Berlin, 2nd edition, 1999. doi:10.1007/3-540-28788-4.

[13] Wolfgang Espelage, Frank Gurski, and Egon Wanke. How to solve np-hard graph problems on clique-width bounded graphs in polynomial time. In Andreas Brandstädt and Van Bang Le, editors, Graph-Theoretic Concepts in Computer Science, 27th International Workshop, WG 2001, Boltenhagen, Germany, June 14-16, 2001, Proceedings, volume 2204 of Lecture 
Notes in Computer Science, pages 117-128. Springer, 2001. doi:10.1007/3-540-45477-2\ $-12$.

[14] Sergei Evdokimov, Ilia N. Ponomarenko, and Gottfried Tinhofer. Forestal algebras and algebraic forests (on a new class of weakly compact graphs). Discret. Math., 225(1-3):149172, 2000. doi:10.1016/S0012-365X (00)00152-7.

[15] Erich Grädel, Phokion G. Kolaitis, Leonid Libkin, Maarten Marx, Joel Spencer, Moshe Y. Vardi, Yde Venema, and Scott Weinstein. Finite Model Theory and Its Applications. Texts in Theoretical Computer Science. An EATCS Series. Springer, 2007. doi:10.1007/ 3-540-68804-8.

[16] Erich Grädel and Martin Otto. Inductive definability with counting on finite structures. In Egon Börger, Gerhard Jäger, Hans Kleine Büning, Simone Martini, and Michael M. Richter, editors, Computer Science Logic, 6th Workshop, CSL '92, San Miniato, Italy, September 28 - October 2, 1992, Selected Papers, volume 702 of Lecture Notes in Computer Science, pages 231-247. Springer, 1992. doi:10.1007/3-540-56992-8\_15.

[17] Martin Grohe. Fixed-point logics on planar graphs. In Thirteenth Annual IEEE Symposium on Logic in Computer Science, Indianapolis, Indiana, USA, June 21-24, 1998, pages 6-15. IEEE Computer Society, 1998. doi:10.1109/LICS.1998.705639.

[18] Martin Grohe. Isomorphism testing for embeddable graphs through definability. In F. Frances Yao and Eugene M. Luks, editors, Proceedings of the Thirty-Second Annual ACM Symposium on Theory of Computing, May 21-23, 2000, Portland, OR, USA, pages 63-72. ACM, 2000. doi:10.1145/335305.335313.

[19] Martin Grohe. Descriptive Complexity, Canonisation, and Definable Graph Structure Theory, volume 47 of Lecture Notes in Logic. Cambridge University Press, 2017. doi: 10.1017/9781139028868.

[20] Martin Grohe and Sandra Kiefer. A linear upper bound on the weisfeiler-leman dimension of graphs of bounded genus. In Christel Baier, Ioannis Chatzigiannakis, Paola Flocchini, and Stefano Leonardi, editors, 46th International Colloquium on Automata, Languages, and Programming, ICALP 2019, July 9-12, 2019, Patras, Greece, volume 132 of LIPIcs, pages 117:1-117:15. Schloss Dagstuhl - Leibniz-Zentrum für Informatik, 2019. doi:10. 4230/LIPICs . ICALP. 2019.117.

[21] Martin Grohe and Julian Mariño. Definability and descriptive complexity on databases of bounded tree-width. In Catriel Beeri and Peter Buneman, editors, Database Theory - ICDT '99, 7th International Conference, Jerusalem, Israel, January 10-12, 1999, Proceedings, volume 1540 of Lecture Notes in Computer Science, pages 70-82. Springer, 1999. doi: $10.1007 / 3-540-49257-7 \backslash \_6$.

[22] Martin Grohe and Dániel Marx. Structure theorem and isomorphism test for graphs with excluded topological subgraphs. SIAM J. Comput., 44(1):114-159, 2015. doi:10.1137/ 120892234.

[23] Martin Grohe, Daniel Neuen, Pascal Schweitzer, and Daniel Wiebking. An improved isomorphism test for bounded-tree-width graphs. ACM Trans. Algorithms, 16(3):34:1-34:31, 2020. doi: $10.1145 / 3382082$.

[24] Martin Grohe and Pascal Schweitzer. Isomorphism testing for graphs of bounded rank width. In Venkatesan Guruswami, editor, IEEE 56th Annual Symposium on Foundations of Computer Science, FOCS 2015, Berkeley, CA, USA, 17-20 October, 2015, pages 10101029. IEEE Computer Society, 2015. doi:10.1109/FOCS.2015.66. 
[25] Martin Grohe and Pascal Schweitzer. Computing with tangles. SIAM J. Discrete Math., 30(2):1213-1247, 2016. doi:10.1137/15M1027565.

[26] Yuri Gurevich. Logic and the challenge of computer science. In Trends in theoretical computer science (Udine, 1984), volume 12 of Principles Comput. Sci. Ser., pages 1-57. Computer Sci. Press, Rockville, MD, 1988.

[27] Yuri Gurevich and Saharon Shelah. Fixed-point extensions of first-order logic. Ann. Pure Appl. Logic, 32:265-280, 1986. doi:10.1016/0168-0072(86)90055-2.

[28] Lauri Hella. Logical hierarchies in PTIME. Inf. Comput., 129(1):1-19, 1996. doi :10.1006/ inco.1996.0070.

[29] Neil Immerman. Languages that capture complexity classes. SIAM J. Comput., 16(4):760778, 1987. doi:10.1137/0216051.

[30] Neil Immerman. Descriptive complexity. Graduate texts in computer science. Springer, 1999. doi:10.1007/978-1-4612-0539-5.

[31] Neil Immerman and Eric Lander. Describing graphs: A first-order approach to graph canonization. In Alan L. Selman, editor, Complexity Theory Retrospective: In Honor of Juris Hartmanis on the Occasion of His Sixtieth Birthday, July 5, 1988, pages 5981. Springer New York, New York, NY, 1990. URL: http://dx.doi.org/10.1007/ 978-1-4612-4478-3_5, doi:10.1007/978-1-4612-4478-3_5.

[32] Sandra Kiefer and Daniel Neuen. The power of the Weisfeiler-Leman algorithm to decompose graphs. SIAM J. Discret. Math., 36(1):252-298, 2022. doi:10.1137/20m1314987.

[33] Sandra Kiefer, Ilia Ponomarenko, and Pascal Schweitzer. The weisfeiler-leman dimension of planar graphs is at most 3. J. ACM, 66(6):44:1-44:31, 2019. doi:10.1145/3333003.

[34] Stephan Kreutzer. Expressive equivalence of least and inflationary fixed-point logic. Ann. Pure Appl. Logic, 130(1-3):61-78, 2004. doi:10.1016/j.apal.2004.02.001.

[35] Leonid Libkin. Elements of Finite Model Theory. Texts in Theoretical Computer Science. An EATCS Series. Springer, 2004. URL: http://www.cs.toronto.edu/\%7Elibkin/fmt, doi : 10.1007/978-3-662-07003-1.

[36] Daniel Lokshtanov, Marcin Pilipczuk, Michal Pilipczuk, and Saket Saurabh. Fixedparameter tractable canonization and isomorphism test for graphs of bounded treewidth. SIAM J. Comput., 46(1):161-189, 2017. doi:10.1137/140999980.

[37] Eugene M. Luks. Isomorphism of graphs of bounded valence can be tested in polynomial time. J. Comput. Syst. Sci., 25(1):42-65, 1982. doi:10.1016/0022-0000(82)90009-5.

[38] Martin Otto. Bounded Variable Logics and Counting: A Study in Finite Models, volume 9 of Lecture Notes in Logic. Cambridge University Press, 2017. doi:10.1017/9781316716878.

[39] Sang-il Oum. Rank-width and vertex-minors. J. Comb. Theory, Ser. B, 95(1):79-100, 2005. doi:10.1016/j.jctb.2005.03.003.

[40] Sang-il Oum. Rank-width is less than or equal to branch-width. Journal of Graph Theory, 57(3):239-244, 2008. doi:10.1002/jgt.20280.

[41] Sang-il Oum and Paul D. Seymour. Approximating clique-width and branch-width. J. Comb. Theory, Ser. B, 96(4):514-528, 2006. doi:10.1016/j.jctb.2005.10.006. 
[42] Ilia N. Ponomarenko. The isomorphism problem for classes of graphs that are invariant with respect to contraction. Zap. Nauchn. Sem. Leningrad. Otdel. Mat. Inst. Steklov. (LOMI), 174(Teor. Slozhn. Vychisl. 3):147-177, 182, 1988. doi:10.1007/BF01098279.

[43] Neil Robertson and Paul D. Seymour. Graph minors. x. obstructions to tree-decomposition. J. Comb. Theory, Ser. B, 52(2):153-190, 1991. doi:10.1016/0095-8956(91)90061-N.

[44] Moshe Y. Vardi. The complexity of relational query languages (extended abstract). In Harry R. Lewis, Barbara B. Simons, Walter A. Burkhard, and Lawrence H. Landweber, editors, Proceedings of the 14th Annual ACM Symposium on Theory of Computing, May 5-7, 1982, San Francisco, California, USA, pages 137-146. ACM, 1982. doi:10.1145/ 800070.802186 .

[45] Boris Weisfeiler and Andrei Leman. The reduction of a graph to canonical form and the algebra which appears therein. NTI, Series 2, 1968. English translation by Grigory Ryabov available at https://www.iti.zcu.cz/w12018/pdf/wl_paper_translation.pdf.

\section{A Canonisation from Weisfeiler-Leman}

In this section we give a proof of Theorem 2.7. Towards this end, an intermediate theorem needs to be proven first.

Let $G$ be a graph. The $k$-dimensional Weisfeiler-Leman algorithm determines orbits of $G$ if, for every graph $H$, every $v \in V(G)$ and every $w \in V(H)$ such that $\chi_{(\infty)}^{G, k}(v, \ldots, v)=$ $\chi_{(\infty)}^{H, k}(w, \ldots, w)$, there is an isomorphism $\varphi: G \cong H$ such that $\varphi(v)=w$.

Theorem A.1. Let $\mathcal{C}$ be a class of graphs such that the $k$-dimensional Weisfeiler-Leman algorithm identifies all (coloured) graphs $G \in \mathcal{C}$. Then the $(k+1)$-dimensional Weisfeiler-Leman algorithm determines orbits of all (coloured) graphs $G \in \mathcal{C}$.

Proof. Let $G \in \mathcal{C}$ and let $H$ be an arbitrary graph. Also let $v \in V(G)$ and $w \in V(H)$ such that $\chi_{(\infty)}^{G, k+1}(v, \ldots, v)=\chi_{(\infty)}^{H, k+1}(w, \ldots, w)$. Then $\left(G, \chi_{G}^{(v)}\right) \simeq_{k}\left(H, \chi_{H}^{(w)}\right)$. Since the $k$-dimensional Weisfeiler-Leman algorithm identifies all graphs $G \in \mathcal{C}$ this implies that $\left(G, \chi_{G}^{(v)}\right) \cong\left(H, \chi_{H}^{(w)}\right)$. So there is an isomorphism $\varphi: G \cong H$ such that $\varphi(v)=w$.

Theorem A.2 (Theorem 2.7 restated). Let $\mathcal{C}$ be a graph class and suppose the $k$-dimensional Weisfeiler-Leman algorithm identifies all coloured graphs in $\mathcal{C}$. Then there is a graph canonisation for $\mathcal{C}$ that can be computed in time $O\left(n^{k+3} \log n\right)$.

Proof. Let $\kappa: \mathcal{C} \rightarrow \mathcal{G}_{\mathbb{N}}$ be the function computed by Algorithm 1. It is first argued that $\kappa$ canonises the graph class $\mathcal{C}$. Let $G \in \mathcal{C}$. Clearly, $\varphi: V(G) \rightarrow[n]: v_{i} \mapsto i$ is an isomorphism from $G$ to $\kappa(G)$.

So let $H \in \mathcal{C}$ be a second graph such that $G \cong H$. Also let $v_{1}, \ldots, v_{n}$ be the sequence of vertices computed by Algorithm 1 for the graph $G$ and let $w_{1}, \ldots, w_{n}$ be the corresponding sequence for $H$. We prove by induction on $i \in\{0, \ldots, n\}$ that there is an isomorphism $\varphi: G \cong H$ such that $\varphi\left(v_{j}\right)=w_{j}$ for all $j \leq i$. The base step $i=0$ is exactly the assumption $G \cong H$. So let $i \geq 1$ and let $\varphi: G \cong H$ such that $\varphi\left(v_{j}\right)=w_{j}$ for all $j \leq i-1$. Then $\left(G, \chi_{G, i}\right) \cong\left(H, \chi_{H, i}\right)$ and $\chi_{G, i}\left(v_{i}\right)=\chi_{H, i}\left(w_{i}\right)$. Since the $(k+1)$-dimensional Weisfeiler-Leman algorithm determines orbits for all graphs $G \in \mathcal{C}$ it follows that there is an isomorphism $\varphi:\left(G, \chi_{G, i}\right) \cong\left(H, \chi_{H, i}\right)$ such that $\varphi\left(v_{i}\right)=w_{i}$. But this isomorphism has to map $v_{j}$ to $w_{j}$ for all $j \leq i$ since they have their own colour in the colouring $\chi_{G, i}$.

By the induction principle, $\varphi: V(G) \rightarrow V(H): v_{i} \mapsto w_{i}$ is an isomorphism from $G$ to $H$. Thus, $\kappa(G)=\kappa(H)$. 


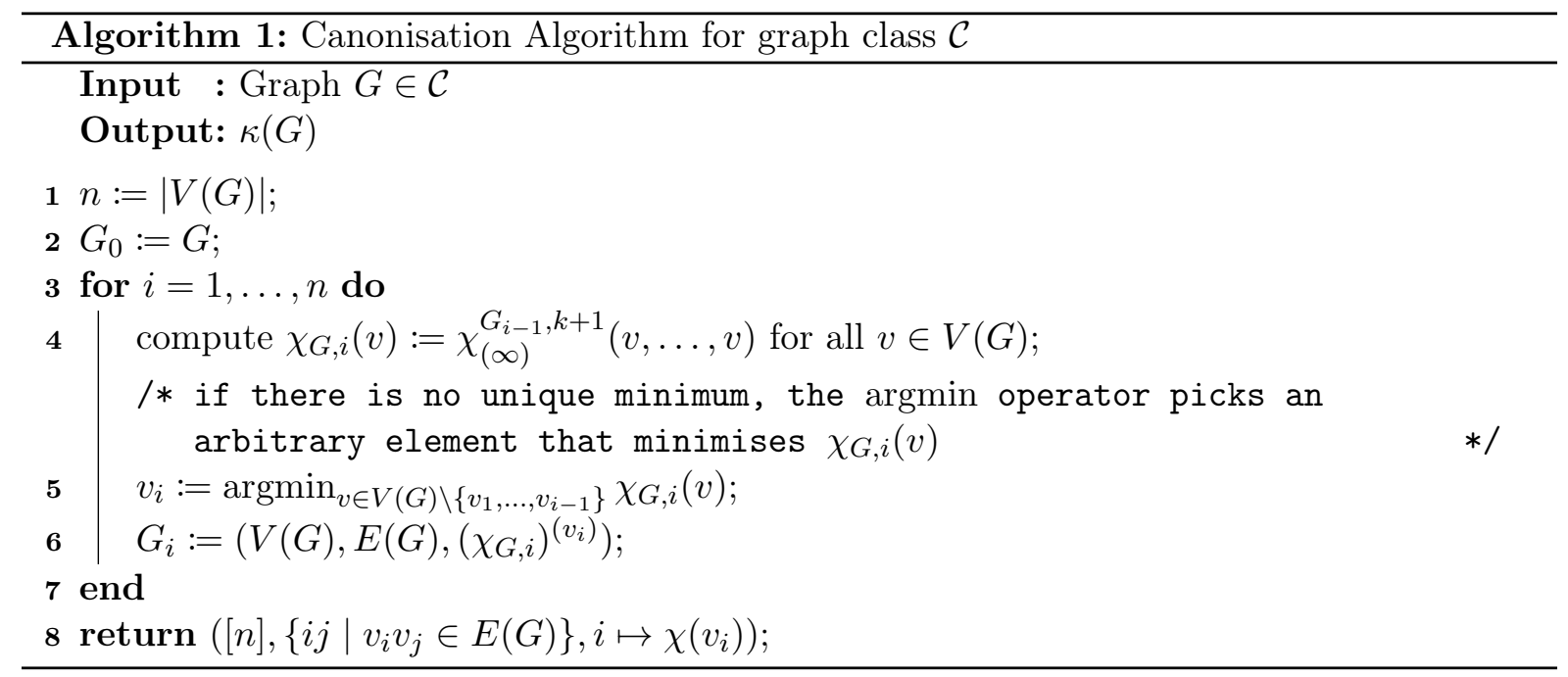

The bound on the running time is immediately clear as the algorithm performs $n$ calls to the $(k+1)$-dimensional Weisfeiler-Leman algorithm, which runs in time $O\left(n^{k+2} \log n\right)$. 